\title{
Heat transfer measurement of turbulent spots in a hypersonic blunt-body boundary layer
}

\author{
By A. FIALA ${ }^{1}$, R. HILLIER ${ }^{1}$, S. G. MALLINSON \\ AND H. S. WIJESIN GHE \\ ${ }^{1}$ Department of Aeronautics, Imperial College, London SW7 2AZ, UK \\ ${ }^{2}$ Faculty of Engineering, University of Technology, Sydney, Australia
}

(Received 7 January 2005 and in revised form 29 November 2005)

\begin{abstract}
This paper presents data on turbulent-spot propagation in the hypersonic boundarylayer flow over a blunted cylindrical body. Data are based on the measurement of time-dependent surface heat transfer rates using gauges positioned as arrays in either the axial or transverse directions. These are used to provide data on individual spots, including sectional profiles, characteristic spot planform geometries, propagation speeds, growth rates and some information on the development of an internal thermal cell structure and corresponding thermal streaks in the base or calm region of the spot.
\end{abstract}

\section{Introduction}

Transition modelling and prediction is a critical topic at hypersonic speeds because of the physically large extent of the transition region and the impact, on narrow design margins, of large variations or uncertainties in heat transfer, skin friction and other flow properties. This present study is concerned with the intermittent region of transition, which extends from the initial breakdown of the laminar layer into turbulent 'spots' up to the position where the boundary layer is fully turbulent. The extent of the intermittent region will be controlled by the rate of production, convection, growth and coalescence of these spots. At hypersonic speeds this length can be substantial; it can easily be greater than the length of the initial laminar flow and could occupy a substantial fraction of a vehicle length. Thus, many critical phenomena, such as severe flow compression (as in an intake for an airbreathing engine), shock wave impingement and flow separation might occur within the intermittent zone.

Spot behaviour has been studied extensively in low-speed flows (see for example, Emmons 1951; Schubaeur \& Klebanoff 1955; Wygnanski, Sokolov \& Friedman 1976; Cantwell, Coles \& Dimotakis 1978; Coles \& Savas 1980; Matsui 1980; Gad-El-Hak, Blackwelder \& Riley 1981; Riley \& Gad-El Hak 1985; Gutmark \& Blackwelder 1987; Sankaran et al. 1991; Gostelow, Hong \& Sheppeard 1992; Hong 1995; Rathnasingham \& Breuer 1997), producing the classical description of an 'arrowhead' planform for a spot. For example, figure 1 presents incompressible spot front and back convection speed data (in a self-similar scaling form), taken using hot-wire anemometry by Wygnanski et al. (1976). With appropriate velocity-time scaling the 'arrowhead' shape contained within the two velocity data lines are similar in shape to the actual spot planform. Experimental data for compressible flows, 


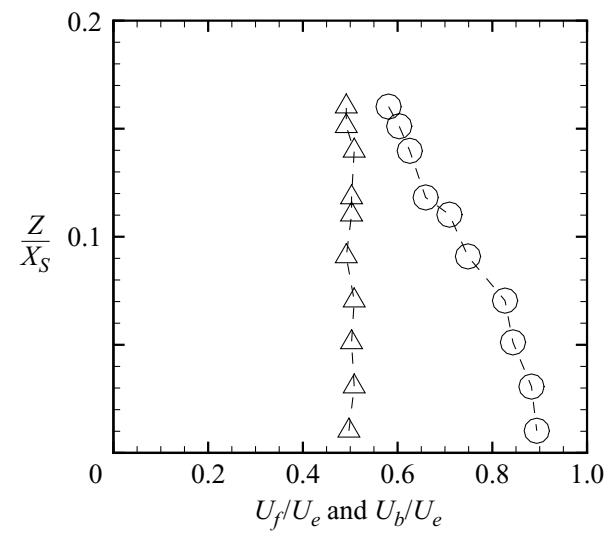

FIGURE 1. Data for propagation speed of spot fronts, $\bigcirc$, and backs, $\triangle$, in incompressible flow, taken from Wygnanski et al. (1976) $X_{S}$ is the distance travelled by the spot front from its origin (spark-generated) and $Z$ is the spanwise distance from the spot centreline.

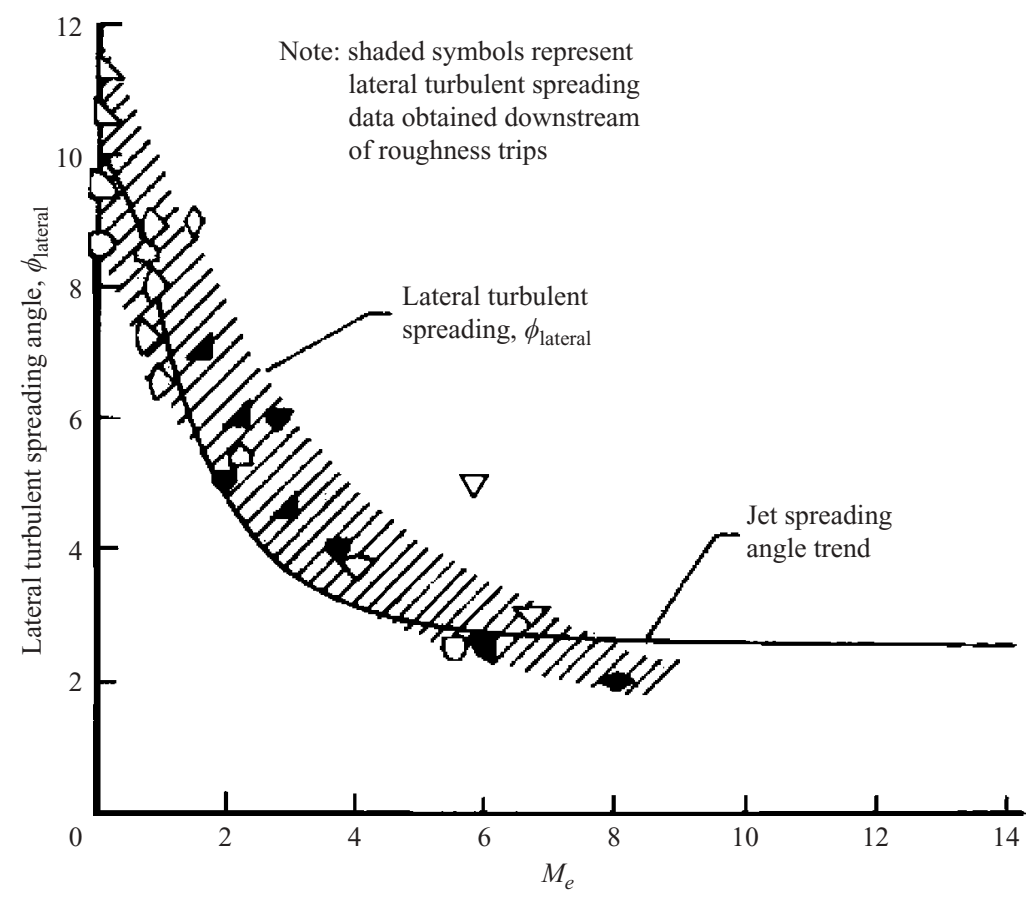

FIGURE 2. Data for dependence of turbulent spot and wedge lateral (spanwise) spreading rates on free-stream Mach number [from Fischer 1972 b].

especially with significant kinetic heating, are much more sparse. Clark, Jones \& LaGraff $(1994,1996)$ present results for transonic flows and studies at hypersonic speeds including Nagamatsu, Sheer \& Graber (1967), Fischer (1972a,b), Zanchetta \& Hillier (1996a, b), Mee \& Goyne (1996), Mee (2001, 2002). Fischer (1972 b) collated available data for spreading rates of turbulent spots and wedges. These are presented in figure 2, and show how the spreading rate tends to reduce with increased Mach number. This reduction, which is also predicted by the theoretical work of Doorly 


\begin{tabular}{lccccc}
\hline$M_{\infty}$ & $\mathrm{d} M / \mathrm{d} x\left(\mathrm{~m}^{-1}\right)$ & $P_{0, \infty}(\mathrm{MPa})$ & $T_{0, \infty}(\mathrm{K})$ & $T_{\text {wall }}(\mathrm{K})$ & $\operatorname{Re}_{\infty}\left(\mathrm{m}^{-1}\right)$ \\
$8.9 \pm 0.05$ & $0.24 \pm 0.03$ & $60.8 \pm 1.2$ & $1150 \pm 45$ & $293 \pm 5$ & $47400000 \pm 6 \%$
\end{tabular}

TABLE 1. Test-section flow conditions.

\& Smith (1992) to give an edge Mach number $\left(M_{e}\right)$ dependence for the half-wake spreading angle of $3^{-3 / 2} 2^{1 / 2} M_{e}^{-1}$, contributes to the elongated intermittent zone at hypersonic speeds. In low speeds, there have been extensive visualization studies of spots, using dyes or smoke. At high speeds, there have only been a few visualization studies, based on shadowgraph or schlieren photographs. Schneider (2004) presents a, previously unpublished, shadowgraph of Reda $(1977,1979)$ for Mach 4.3 flow past a sharp cone. These images show edge views of turbulent spots within a laminar flow, and include the associated pressure waves from the leading and trailing edges of the spot, which enabled the relative, or convective, speeds of the spots to be determined. James (1958) also used shadowgraph to detect turbulent spots in tests on slender bodies in a free-flight facility, for Mach numbers from 2.7 to 10 . Using simultaneous orthogonal shadowgraph, he was able to extract some planform data for well-developed spots, whose axial scales were two or more orders of magnitude greater than the displacement thickness of the surrounding laminar boundary layer. This produced 'arrowhead' spot planforms that were broadly similar to those implied by figure 1. Other studies of spots in high-speed flows have been based on surface heat transfer measurements, using rapid-response sensors (e.g. Nagamatsu et al. 1967; Zanchetta \& Hillier (1996a,b), Mee \& Goyne 1996; Mee 2001, 2002). These have produced spot leading-edge and trailing-edge convection speeds comparable to those in figure 1, but with no overall detail on planform shapes.

The particular driving force behind this study was an earlier experiment at Imperial College by Zanchetta \& Hillier $(1996 a, b)$ on blunted, $5^{\circ}$ semi-angle cones. It has long been known (e.g. Stetson \& Rushton 1967) that, at hypersonic speeds, initial blunting of cones causes a very substantial progressive delay in the onset of transition, but that, at sufficiently large nose radii (or, more precisely, nose radius Reynolds numbers), early transition occurs again. This phenomenon is often called 'transition reversal'. The mechanism of transition reversal is not fully understood, but it is clearly linked with the effect of the entropy layer - the region of low total pressure and low unit Reynolds number generated by the bow shock wave - on the development of the boundary layer. Zanchetta \& Hillier $(1996 a, b)$ found in their experiments that for nose radius Reynolds numbers less than about 200000 (a $4 \mathrm{~mm}$ nose radius using the test conditions of table 1) transition distances rapidly increased with increased nose blunting, with little apparent sensitivity to surface roughness. Above this nose radius, however, transition lengths were further increased with increased nose blunting, but now the flow became sensitive to surface roughness near the spherical nose/cone frustrum junction so that early transition was also possible. Depending upon the model surface condition, tests with a $25 \mathrm{~mm}$ nose radius (nose radius Reynolds number of 1.2 million) showed that it was possible to have fully turbulent flow from the sphere/frustrum junction, or fully laminar flow over the whole model length (a chord Reynolds number of 30 million based upon free-stream conditions), or an intermittently turbulent flow.

Generally, measurement and resolution of time-dependent data is very demanding in high-speed flows. In the Imperial College gun tunnel (see $§ 2$ ), for example, significant 


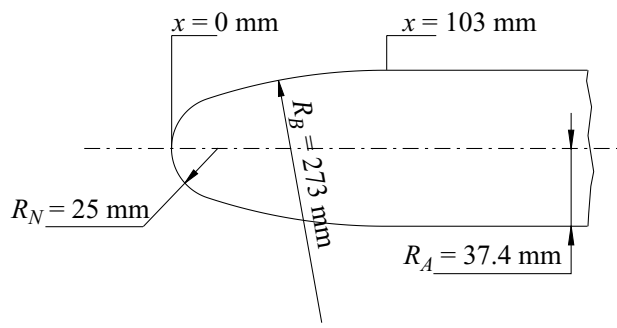

FIGURE 3. Schematic of model nose section, comprising a spherical nose, with radius, $R_{N}$, of $25 \mathrm{~mm}$, a cylindrical afterbody with radius, $R_{A}$, of $37.4 \mathrm{~mm}$ and blending section with constant arc radius, $R_{B}$, of $273 \mathrm{~mm}$. The cylindrical section starts at $x=103 \mathrm{~mm}$ and extends to $650 \mathrm{~mm}$.

turbulence energy can be expected at frequencies up to at least $500 \mathrm{kHz}$, well beyond the $125 \mathrm{kHz}$ sampling rates of the installed data system. The blunt-cone study, however, showed that, with careful selection of the configuration and test conditions, a systematic study of well-developed spots was possible. This arose from the observation that the spot formation rate was low and that for chordwise positions of 400-600 mm it was possible to obtain isolated spots with characteristic streamwise scales in excess of $100 \mathrm{~mm}$. At such a scale, and with convection speeds less than $1000 \mathrm{~m} \mathrm{~s}^{-1}$, the spatial and temporal resolution available would enable a good picture to be developed for the overall spot characteristics. The design concept for the test model was therefore configured around accumulating test data at large chord lengths, for a design expected to produce low spot-production rates. Surface heat transfer monitoring of turbulent spot activity requires a dense coverage of surface instrumentation, in both the axial and circumferential directions, and instrumentation is expensive. To minimize this effort, it was decided that a blunted circular cylinder (at zero incidence) would provide the best configuration. With careful modular construction, this permits a single instrumentation unit to be successively located at various chordwise stations. This is a substantial advantage over the cone configuration, which requires separate instrumentation modules to be produced for each desired measurement station.

\section{Experimental details}

\subsection{Gun tunnel facility}

The experiments were conducted in the Imperial College gun tunnel, using nitrogen as the test gas. The tunnel has an axisymmetric nozzle, providing a test 'diamond' of the order of $1.5 \mathrm{~m}$ long, which permits the testing of large-chord slender models at high unit Reynolds numbers. The total flow duration time for a single firing is $20 \mathrm{~ms}$, with a sample window of approximately $6 \mathrm{~ms}$. A detailed calibration of the tunnel by Mallinson et al. (2000) shows a highly axisymmetric flow, with weak axial gradients of order $2.2 \%$ per metre in Mach number. All flow properties have been evaluated at the nominal calibration conditions given in table 1 and the CFD modelling reproduces the best possible conditions by incorporating the calibrated tunnel gradient. The Prandtl number, $P r$, is assumed constant at 0.72 , and the viscosity for nitrogen is evaluated using the expression given by Keyes (1952).

\subsection{Model and instrumentation}

The basic test model comprised a $650 \mathrm{~mm}$ long, cylindrical ( $37.4 \mathrm{~mm}$ radius) afterbody and a $25 \mathrm{~mm}$ radius spherical nose (figure 3 ). This nose radius was the same as that 
of the earlier study on blunted cones, by Zanchetta \& Hillier $(1996 a, b)$, and the afterbody diameter was determined by the requirement that the expected spanwise scale of an individual turbulent spot should not occupy too large a proportion of the model circumference. The cylindrical afterbody and spherical nose were blended by a $273 \mathrm{~mm}$ radius arc, such that continuity of surface slope was maintained; this results in discontinuities in surface pressure gradient although a continuous, favourable streamwise pressure gradient is maintained everywhere along the model length as shown by the computational fluid dynamics (CFD) simulations presented in figure 7. The total axial length from the stagnation point to the junction between the blending arc and the cylindrical afterbody is $103 \mathrm{~mm}$.

The model was constructed from a series of different interchangeable segments, which allowed measurements to be made at a number of streamwise locations using a single instrumentation module. Careful manufacturing, and machining of the fully assembled model, was used to provide smooth continuity from one model segment to the next. Heat transfer modules comprised thin-film platinum resistance gauges, handpainted onto machinable ceramic (MACOR) blocks. These blocks had previously been machined integral with the basic model to provide excellent surface continuity. Two different types of module were employed. The first comprised gauges spaced along the surface in the axial direction to provide streamwise distributions of heat transfer, using a maximum gauge pitch axially of $4 \mathrm{~mm}$. The second type of module consisted of 18 sensors spaced at $4 \mathrm{~mm}$ ( or $6.1^{\circ}$ ) pitch around the circumference. Assembled details for these are shown in figure 4. The gauge spacing, axial and circumferential, provided simultaneous sampling of all gauges over a domain comparable to the expected turbulent spot size. The characteristic spatial resolution of individual sensors was approximately $2.5 \mathrm{~mm}$. The axial modules could be positioned at various locations along the model chord, providing full coverage over the range $125 \mathrm{~mm} \leqslant x \leqslant 520 \mathrm{~mm}$. The circumferential array could be located at discrete axial positions and in this study, gauge module positions at $x=213 \mathrm{~mm}, 279 \mathrm{~mm}$ and $347 \mathrm{~mm}$ were chosen. Because of design and instrumentation restrictions it was not possible to operate axial and circumferential sensor arrays simultaneously.

The resistance-temperature relation for the platinum films was obtained by waterbath calibration. The thermal product $\sqrt{(\rho c k)}(\rho=$ density, $c=$ specific heat, $k=$ thermal conductivity) for MACOR was taken as $2010 \mathrm{~J} \mathrm{~m}^{-2} \mathrm{~K}^{-1} \mathrm{~s}^{-0.5}$ at $290 \mathrm{~K}$, based on direct calibration (Zanchetta 1996). Sensor frequency response was assessed using the formulae developed in Schultz \& Jones (1973). With thin-film techniques, the surface heat transfer rate is obtained by integration of the inferred substrate (MACOR) surface-temperature history which is not exactly the same as the effective temperature registered by the surface thin-film (platinum) resistance-thermometer, because of the finite film thickness. Based on the average platinum film thickness of $0.35 \mu \mathrm{m}$ and the properties of the MACOR substrate, the gauges would register $90 \%$ of a step change in surface heat transfer in $11 \mu \mathrm{s}$, according to Schultz \& Jones (1973). Gauge erosion is minimal, so that all gauges survived the complete test programme, with only minor resistance changes.

The transient capture system is triggered by the total pressure signal at the nozzle throat when the tunnel fires. The analogue outputs from the gauges are amplified and low-pass filtered at $45 \mathrm{kHz}$ before being digitized by a 12-bit analogue-to-digital converter at a sample rate of $125 \mathrm{kHz}$ for each channel. The digitized signals are then downloaded to a PC system and integrated to provide heat transfer rates using the algorithm due to Cook \& Felderman (1966) (also see Schultz \& Jones 1973). 


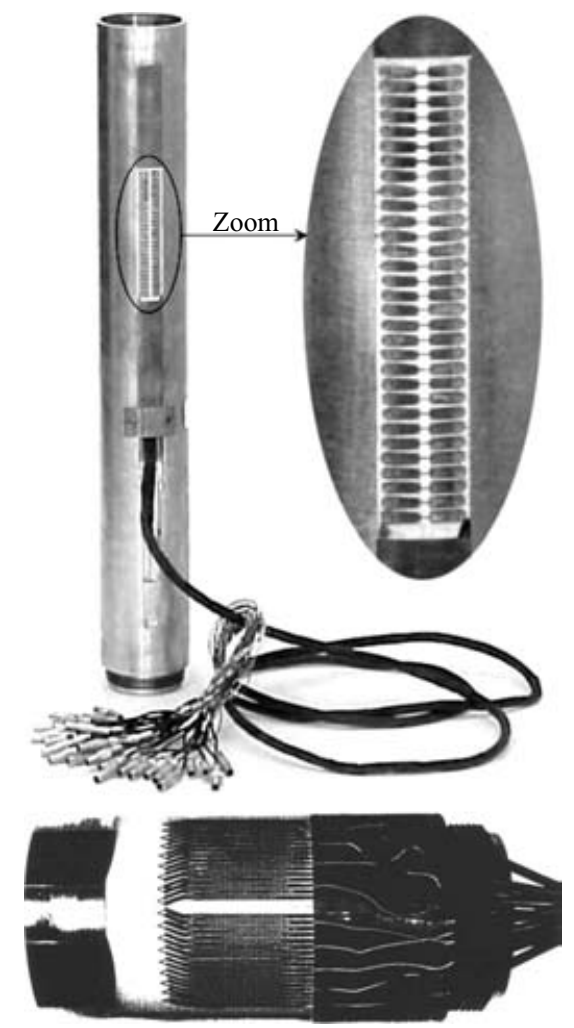

FIGURE 4. Instrumented model segments for both axial and circumferential heat transfer array measurement. In the axial array the thin-film sensors comprise the fine central line; the thicker outboard lines are low resistance gold paint connectors. For the circumferential array, the platinum sensor is the V-shape.

\section{CFD modelling}

\subsection{CFD modelling and mesh resolution}

Experimental data are confined to time-dependent heat transfer rate measurements on the surface and these are accumulated on the cylindrical body, in the interval $125 \mathrm{~mm} \leqslant x \leqslant 520 \mathrm{~mm}$. Other data required in the reduction process or in interpreting the experiments, in particular reference surface heat transfer distributions and estimates of boundary-layer thicknesses, have been determined from CFD modelling. The primary reference quantities required are laminar so that the following CFD comments, unless otherwise stated, relate specifically to the laminar simulation. The numerical procedure has been described elsewhere (Hillier, Kirk \& Soltani 1995; Jackson, Hillier \& Soltani 2001; Hillier et al. 2003), and is formulated here as a second-order accurate 'convection-diffusion-split' axisymmetric Navier-Stokes code. The fluxes for the convective, or Euler part, are solved using the explicit generalized Riemann problem of Ben-Artzi \& Falcovitz (1984) whilst the diffusive, or viscous, fluxes are evaluated by an implicit centred-differencing procedure.

The computations are carried out using a structured mesh that extends up to $550 \mathrm{~mm}$ chord in the streamwise direction and sufficiently far from the body surface that the outer mesh boundary is everywhere positioned outboard of the bow shock wave. Free-stream boundary values are imposed at this outer boundary, taken from 

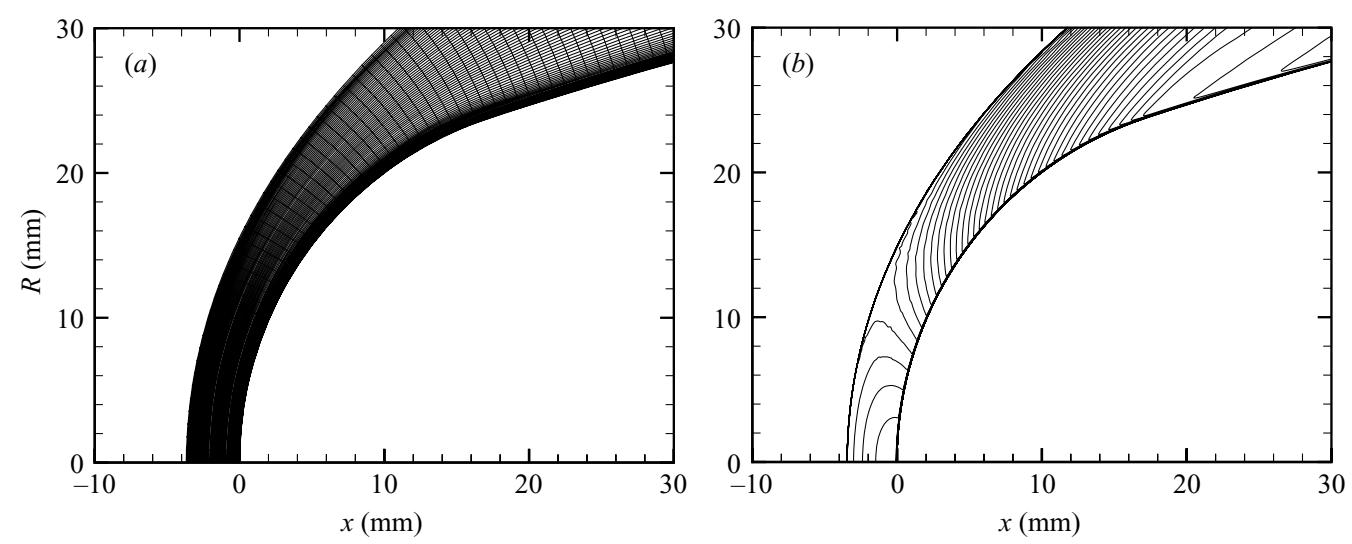

FiguRE 5. (a) Detail of the computational mesh in the region of the model nose for the coarsest mesh simulation. Medium and fine meshes correspond to successive halving of cell sizes in both coordinate directions. (b) Density contours (increments of 0.125 in $\Delta \rho / \rho_{\infty}$ ) for the laminar simulation using the finest mesh.

the tunnel calibration referred to in $\S 2.1$ and including the weak axial gradients discussed earlier (also see Mallinson et al. 2000). The mesh was also developed adaptively, to provide high-resolution capture of both the shock wave and also the boundary layer. This process enforced a pre-specified number of cells from the body surface to the boundary-layer edge and to the bow shock wave, together with a mesh-stretching procedure that provided a further local clustering of cells near the bow shock wave and managed the cell size distribution within the boundary layer. A detailed view of the mesh in the immediate nose region is illustrated in figure 5( $a$ ), for the coarsest mesh simulation. For the finest mesh simulations (the mesh is not shown, simply because of the density of mesh lines), the converged solution corresponded to 800 cells over the $550 \mathrm{~mm}$ axial length of the geometry, $300( \pm 0.25)$ cells from the body surface to the shock wave (for all axial locations) and $40( \pm 1)$ cells from the body surface to the boundary-layer edge. Figure $5(b)$ shows the resultant density contours in the nose region for this finest mesh simulation. The viscous layer in the region of the stagnation point is so thin, approximately $0.1 \mathrm{~mm}$ for $\delta_{0.99}$ compared with the $25 \mathrm{~mm}$ nose radius, that it is barely visible. A larger domain of the computed flow field, extending to $x=181 \mathrm{~mm}$, is shown in figure 6 , where the upper and lower halves of the picture are, respectively, the CFD density contours and an experimental schlieren visualization. The basic bow shock wave shape agrees very well between experiment and CFD, but it is difficult to extract much interior detail from the experimental visualization. Identifying the boundary layer itself is difficult. This is, in part, because it is very thin, as also seen in the CFD modelling. It is also because of three other effects: the combination of low background density which reduces the optical sensitivity; the 'circumferential' nature of the test surface that reduces schlieren contrast compared with a planar configuration; and the entropy-layer effect, which results in the boundary-layer edge density and the wall density being virtually the same. The cause of the disturbances or striations in the shock layer is not clear and it is difficult to isolate the contaminating optical effect that arises from the test section flow being an axisymmetric free jet, confined within a larger closed test section. The shear layer at the jet boundary is turbulent and this turbulence is then seen in the schlieren visualization, although the highly swept Mach lines of the test flow mean 


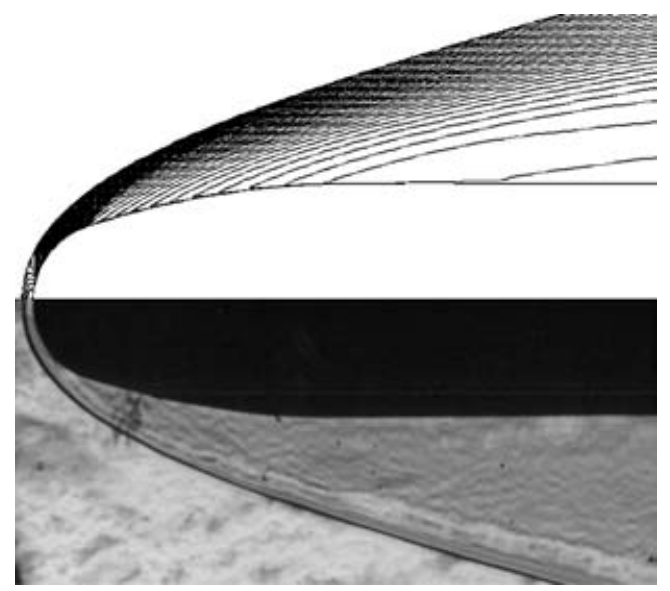

FIGURE 6. Comparison between CFD density contours and schlieren visualization.
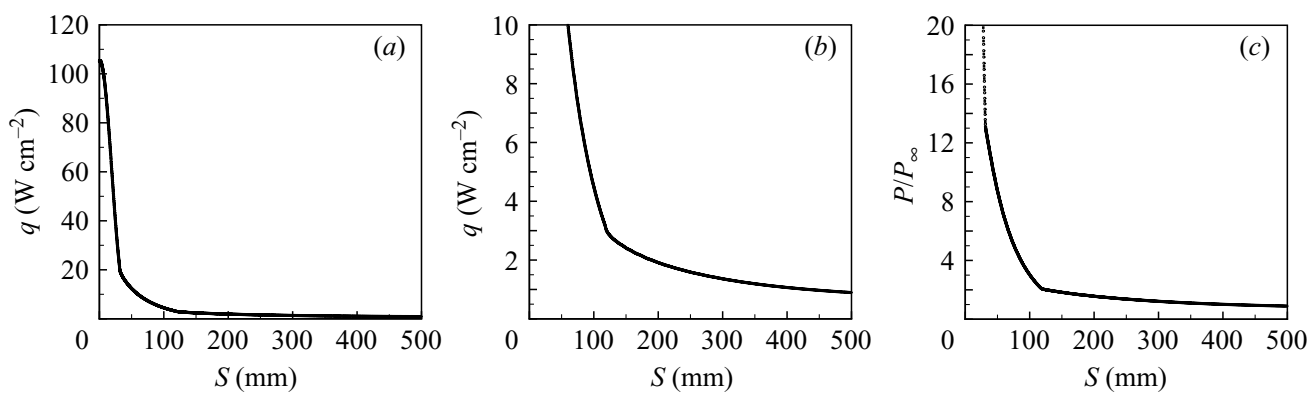

FIGURE 7. $(a, b)$ Variation of computed laminar heat transfer with wetted distance $(s)$ along the body surface. The medium and fine meshes represent global changes of 2:1 in cell linear dimensions. (c) Computed pressure distribution. Because the medium and fine solutions are virtually indistinguishable, the symbols are not separately identified.

that disturbances are unable to radiate onto the test model. For all these reasons, optical visualization of turbulent spots was not possible

At the finest level of mesh resolution, the computed solution can be regarded as mesh-converged. Figures $7(a)$ and $7(b)$, for example, show the variation of computed heat transfer rate (shown at two different ordinate scales to indicate the full detail) versus wetted distance along the body surface from the stagnation point. The heat transfer is the critical property from the viewpoint of this study and also serves as a sensitive indicator of mesh refinement. In this case, both fine and medium mesh solutions are included, but are indistinguishable from each other; all later CFD data correspond to the finest mesh. The effect of the tunnel axial gradient, which is included in the CFD simulations, has been to reduce the heat transfer rate at the $500 \mathrm{~mm}$ position by $7 \%$ compared with a zero gradient simulation. Figure $7(c)$ presents the corresponding distribution of computed surface pressure, which indicates that the test boundary-layer development will be under a continuous favourable pressure gradient. The gradient discontinuities seen in the heat transfer and pressure distributions correspond to the two matching points, between the blending profile and the spherical nose and the cylindrical afterbody, respectively, where there are discontinuities in body streamwise curvature. 


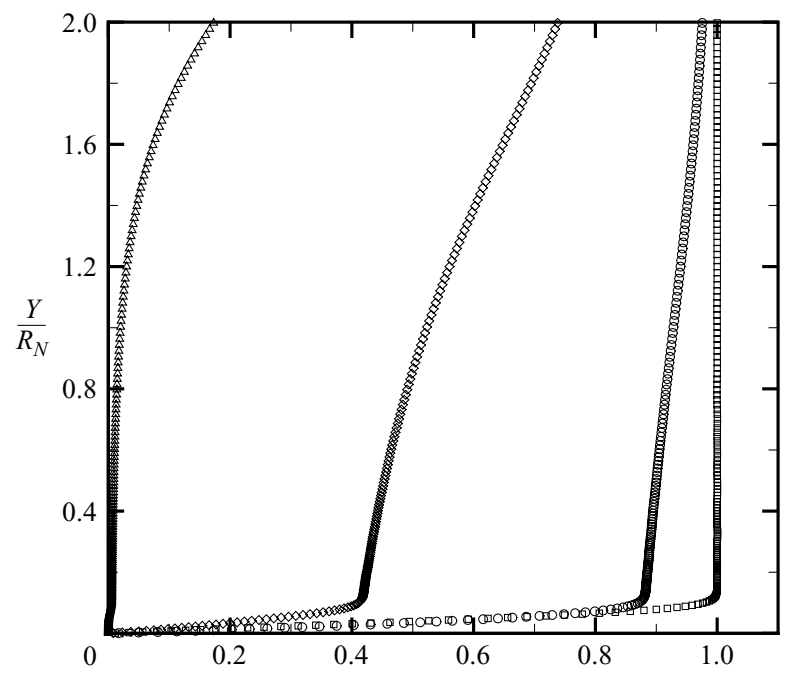

FIGURE 8. Data profiles, in direction $Y$ normal to the body surface (normalized by the nose radius $\left.R_{N}\right)$ at $x=400 \mathrm{~mm} . \Delta, P_{0} / P_{0 \infty} ; \diamond, M / M_{\infty} ; \bigcirc, U / U_{\infty} ; \square,\left(T_{0}-T_{w}\right) /\left(T_{0 \infty}-T_{w}\right)$.

$\begin{array}{lccccc}x \text {-position }(\mathrm{mm}) & 213 & 279 & 347 & 450 & 521 \\ \delta_{1, L}(\mathrm{~mm}) & 0.61 & 0.78 & 0.947 & 1.18 & 1.32 \\ \delta_{2, L}(\mathrm{~mm}) & 0.194 & 0.233 & 0.268 & 0.313 & 0.338 \\ M_{e} & 3.43 & 3.54 & 3.62 & 3.71 & 3.74 \\ U_{e} / U_{\infty} & 0.863 & 0.870 & 0.876 & 0.882 & 0.884 \\ R e_{e}\left(\mathrm{~m}^{-1}\right) & 3270000 & 3060000 & 2910000 & 2760000 & 2690000 \\ P_{0 e} / P_{0 \infty}(\%) & 0.579 & 0.580 & 0.580 & 0.581 & 0.581 \\ \Lambda & -0.23 & -0.28 & -0.28 & -0.25 & -0.24\end{array}$

TABLE 2. CFD prediction for laminar boundary-layer parameters at specific stations.

\subsection{The entropy layer, boundary-layer edge conditions and integral thicknesses}

Defining the boundary-layer edge usually has a special complication for hypersonic blunt-body flows, because the rotational entropy layer formed by the curved bow shock wave means that the boundary layer does not asymptote to a clearly defined zero vorticity or constant total pressure edge condition. Figure 8 illustrates this by presenting CFD profile data at $x=400 \mathrm{~mm}$, which is a location central to the main region of heat transfer measurement; it should be noted that in terms of overall flow-field scales, the maximum normal distance on this figure, $Y / R_{N}=2$, is just under $50 \%$ of the distance from the body surface to the shock wave. The boundary-layer region is easily identified in figure 8 , however, because it is so thin compared with the entropy layer. A thermal thickness definition $\delta_{T}$ is used here to identify edge conditions, since the total temperature $T_{0}$ must recover to the free-stream value, $T_{0, \infty}$, outside the viscous layer. This thermal thickness is defined as the normal distance from the surface at which $\left(T_{0}-T_{0, \text { wall }}\right) /\left(T_{0, \infty}-T_{0, \text { wall }}\right)=0.995$. Figure 9 shows the resultant variation of the boundary-layer edge values $U_{e} / U_{\infty}$ and $M_{e} / M_{\infty}$ with axial distance and table 2 presents a wider selection of important edge values at the specific $x$-locations that are used later when discussing the experiments. Table 2 shows that the total pressure barely changes over the whole axial length, being less than $0.6 \%$ 


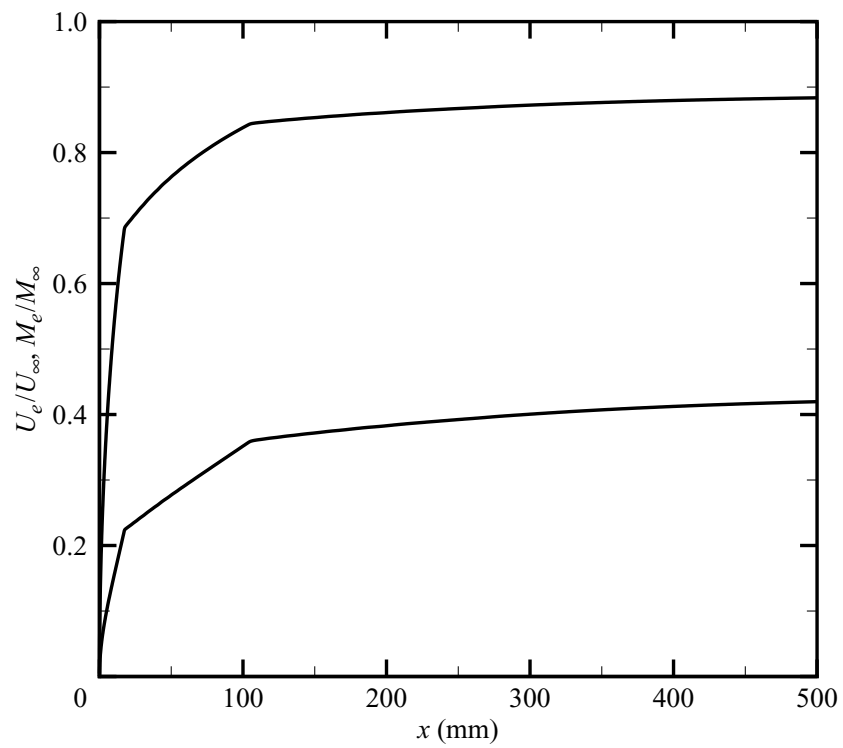

FIGURE 9. Data profiles along boundary-layer edge for $U_{e} / U_{\infty}$ (upper line) and $M_{e} / M_{\infty}$ (lower line).

of the free-stream value, indicating that the edge conditions are controlled by the streamlines that pass through the very central regions of the bow shock wave. In other words, the boundary layer has barely encroached upon the rotational zone generated by the curved bow shock wave and the entropy layer is far from being 'swallowed'. Thus, at the farthest downstream location, the edge velocity, Mach number and unit Reynolds number, respectively, are $0.884 U_{\infty}, 3.74$ (compared with 8.9 for $M_{\infty}$ ) and 2.69 million per metre (compared with 47.4 million per metre for $R e_{\infty}$ ). Table 2 also includes data for the computed variation of the laminar boundary-layer displacement $\left(\delta_{1, L}\right)$ and momentum $\left(\delta_{2, L}\right)$ thicknesses on the cylindrical part of the body, using (see, for example Fernholz \& Finley 1977):

$$
\begin{gathered}
\delta_{1}+\frac{\delta_{1}^{2}}{2 R_{A}}=\int_{0}^{\delta_{\mathrm{T}}}\left(1-\frac{\rho U}{\rho_{\delta_{T}} U_{\delta_{T}}}\right)\left(1+\frac{y}{R_{A}}\right) \mathrm{d} y, \\
\delta_{2}+\frac{\delta_{2}^{2}}{2 R_{A}}=\int_{0}^{\delta_{T}} \frac{\rho U}{\rho_{\delta_{T}} U_{\delta_{T}}}\left(1-\frac{U}{U_{\delta_{T}}}\right)\left(1+\frac{y}{R_{A}}\right) \mathrm{d} y .
\end{gathered}
$$

The 'correction' for axisymmetry in the formulae, given by the second term on the left-hand side of (3.1) and (3.2), is very modest, the laminar displacement thickness $\delta_{1, L}$ amounting to only $1 \mathrm{~mm}$ (compared with the cylinder body radius, $R_{A}=37.4 \mathrm{~mm}$ ) at the $x=400 \mathrm{~mm}$ position. The table also includes the non-dimensional axial pressure gradient parameter, $\Lambda$, suggested by Laderman (1980):

$$
\Lambda=\frac{\delta_{1, L}}{\tau_{w, L}} \frac{\mathrm{d} p}{\mathrm{~d} x},
$$

where the displacement thickness and wall shear stress, $\tau_{w, L}$, are both obtained from the local laminar CFD solution. Kimmel (1997) has indicated that the spot growth and coalescence region is reduced in length by a favourable pressure gradient, although in Kimmel's study the pressure gradient (on an axisymmetric body) was generated 


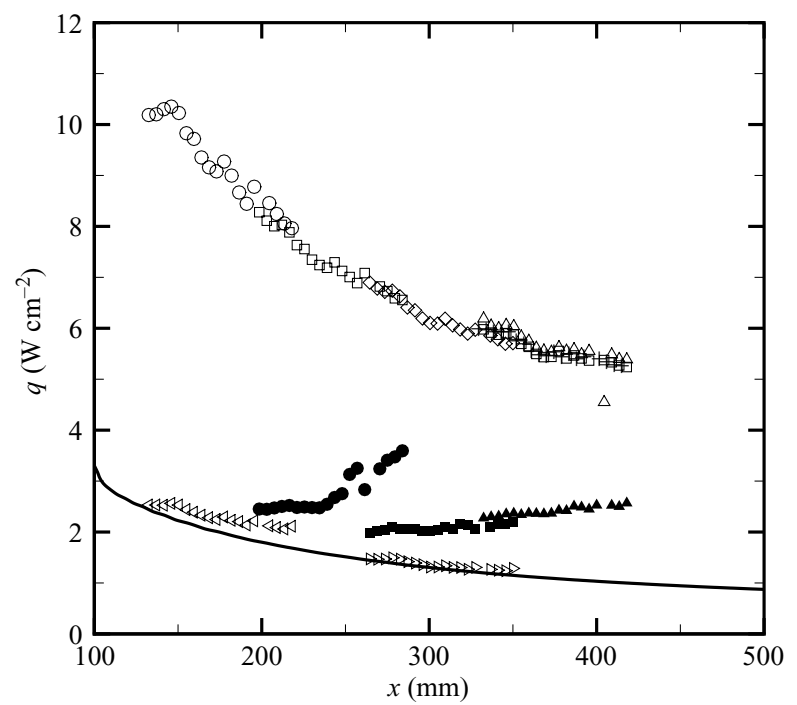

Figure 10. Comparison between laminar CFD (solid line) and time-averaged surface heat transfer for measurements in the range $132 \mathrm{~mm} \leqslant x \leqslant 418 \mathrm{~mm}$. The different symbols represent different experimental runs.

by streamwise body curvature so that the effect of a pure pressure gradient was compounded by streamwise curvature and by streamwise changes of cross-sectional area.

\section{Results}

\subsection{The basic heat transfer signals and intermittency}

For fully laminar or fully turbulent flows, the steady flow window of $6 \mathrm{~ms}$ provides sufficient time for a stationary data sample for time-averaged heat transfer. This window is equivalent to flow convection distances of several thousand times the local boundary-layer thickness. The low spot-production rates that identified this as an attractive experiment, however, mean that a single sample of $6 \mathrm{~ms}$ is not necessarily a sufficient recording time to accumulate stationary data for intermittent flows, but that single runs clearly can be used to provide data on individual intermittent events. Figures 10 and 11(a) present two sets of heat transfer data, each time-averaged over the $6 \mathrm{~ms}$ window, as a distribution against the axial distance, $x$. These figures include the predictions for heat transfer from the laminar flow CFD simulations.

Figure 10 comprises eleven individual runs. The lowest value data sets $(\triangleleft, \triangleright)$, spanning the range $132 \mathrm{~mm} \leqslant x \leqslant 350 \mathrm{~mm}$, were taken with a highly polished model (surface finish $\leqslant 0.25 \mu \mathrm{m}$ ) and clearly are in very close agreement with the laminar predictions. The upper data set, comprising six separate runs and spanning the interval $132 \mathrm{~mm} \leqslant x \leqslant 418 \mathrm{~mm}$, was obtained after deliberate roughening of the nose region of the model (surface finish $\geqslant 2.0 \mu \mathrm{m}$ ) and correspond to a fully turbulent CFD state. The remaining three data sets in figure 10 correspond to an intermittent state, again for a polished model (surface finish $\approx 0.5 \mu \mathrm{m}$ ), with time-averaged levels partway between the laminar and turbulent predictions and with an obvious progressive increase, with downstream distance, in heat transfer (and hence intermittency) for each data set. The lack of consistency between these three sets is probably an indication that the 

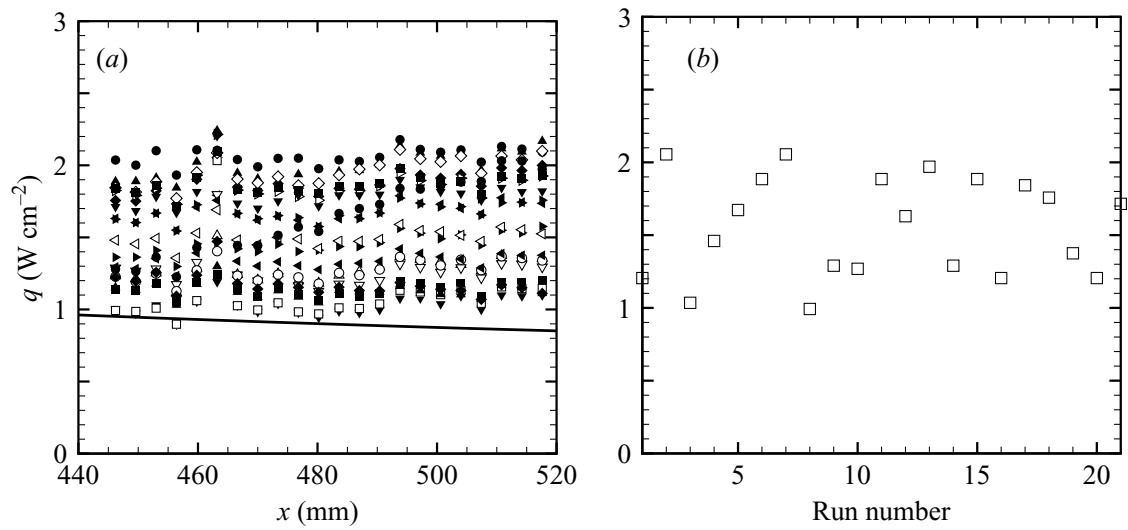

Figure 11. (a) Comparison between laminar CFD and time-averaged surface heat transfer for the range $445 \mathrm{~mm} \leqslant x \leqslant 520 \mathrm{~mm} ;(b)$ variation with run number of averaged heat transfer at $x=480 \mathrm{~mm}$.

individual data records are too short to provide stationary data samples. Figure 11(a) therefore extends the sample record by presenting 21 repeat tests $(6 \mathrm{~ms}$ time-averages in each case), now for a fixed sensor array covering the range $445 \mathrm{~mm} \leqslant x \leqslant 520 \mathrm{~mm}$ and with the model maintained in a polished surface condition. These data also correspond to an intermittent state, occupying the lower $25 \%$ of the range between the laminar CFD $(0.85 \leqslant q \leqslant 1.0)$ and the fully turbulent $(5.0 \leqslant q \leqslant 5.5)$ data (values given in $\mathrm{W} \mathrm{cm}^{-2}$ ). Although the heat transfer level clearly changes from one run to the next, there is, in fact, no systematic variation between runs. This is shown in figure 11(b) where the $6 \mathrm{~ms}$ time-average heat flux values, at the $x=480 \mathrm{~mm}$ location from figure 11(a), are plotted against run number. The lack of any trend shows that there has been no progressive change, such as accumulation of damage to the model, that might otherwise provoke alterations in the transition behaviour. However, the run-to-run variation still indicates that each individual run is too short to obtain stationary mean data for low intermittency signals. These data files are, however, later combined to extract some average statistical data.

Some typical heat transfer time histories for individual gauges are presented in figure 12, all recorded simultaneously in a single run over a $6 \mathrm{~ms}$ window. These correspond to the most downstream intermittent data set of figure 10, with three positions selected at the front $(x=332.5 \mathrm{~mm})$, mid-point $(x=373 \mathrm{~mm})$ and end $(x=418 \mathrm{~mm})$, respectively, of the sensor array. These signals represent the passage of turbulent spots. It is readily seen how the turbulent bursts match between the three sensor positions, with the slight time delays between the bursts corresponding to the spot convection speed that will be discussed later. Various spot events can be seen in figure 12. Event A, for example, shows the growth of a 'new' spike in heat transfer, although it is not possible to determine whether this is the genuine formation of a new spot or the signal generated by a spot sweeping alongside the sensor array and gradually, through its spanwise growth, encroaching upon it. Event B is (probably) an isolated spot, showing the rapid increase at the spot front (the low-time side) and the much more gradual reduction in heat transfer in the base or calm region (high-time side; $q \leqslant 2 \mathrm{~W} \mathrm{~cm}^{-2}$ ). Events $\mathrm{C}, \mathrm{D}$ and $\mathrm{E}$ are the coalescence between two, or more, turbulent events. 

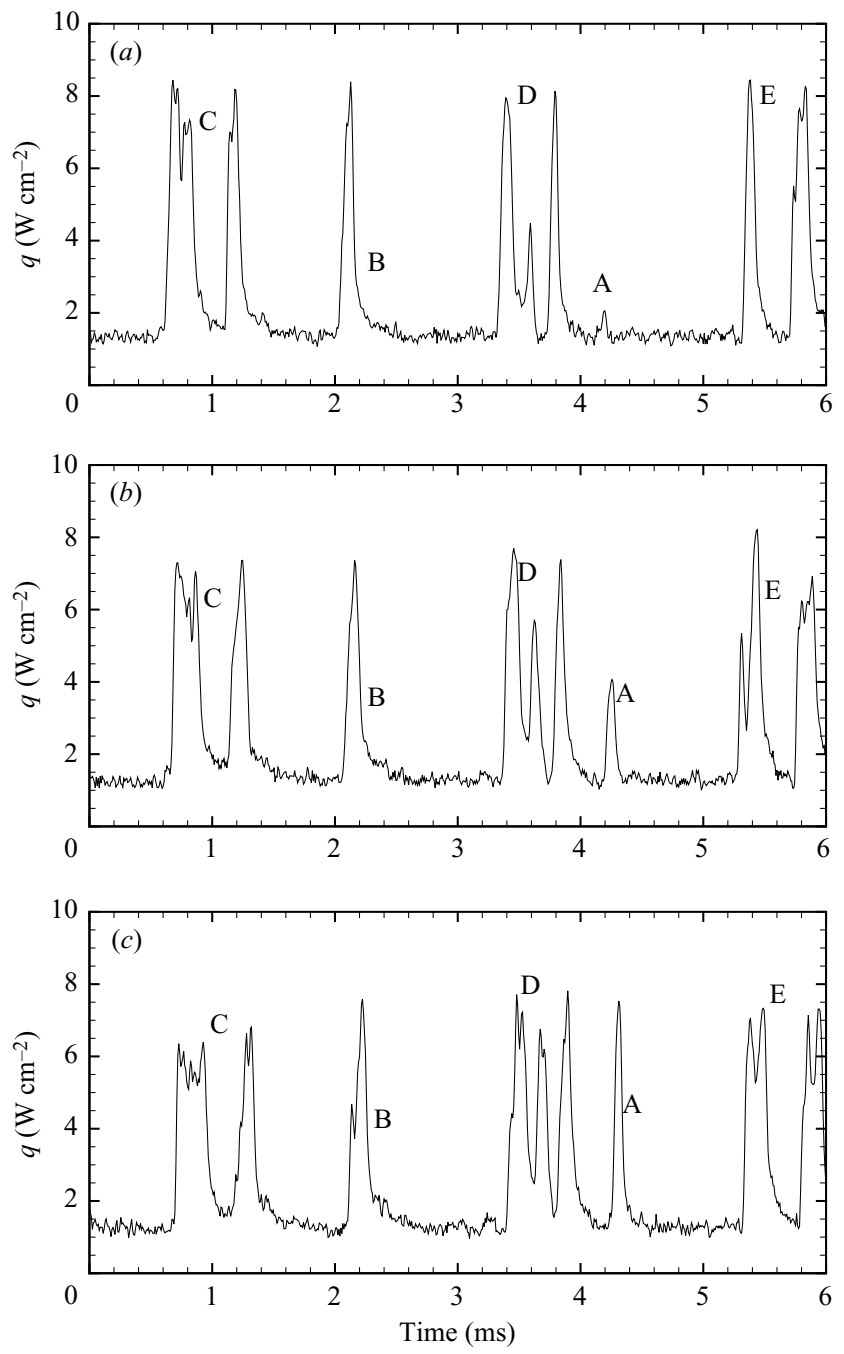

FIGURE 12. Three simultaneous heat transfer time-histories recorded in the intermittent region from the dataset in the range $332.5 \mathrm{~mm} \leqslant x \leqslant 418 \mathrm{~mm}$ of figure 10. (a) Axial location, $x=332.5 \mathrm{~mm}$, (b) $373 \mathrm{~mm}$ and (c) $418 \mathrm{~mm}$.

An alternative form of presentation is to plot the surface heat transfer rate against axial distance, at a given time, essentially providing an instantaneous axial profile through an intermittent event. One such example is shown in figure 13(a) for the emerging A event in figure 12. Three profiles are plotted, at approximately $0.024 \mathrm{~ms}$ intervals, showing a growth (both in magnitude and scale) which reflects both the 'emerging' nature of the event as well as its convection in the flow direction. The streamwise scale is large, however, so that it is clearly difficult to contain the events on the $90 \mathrm{~mm}$ length of the instrumentation module. Even for the most forward sensor position in figure 10, the spot streamwise scales are significant compared with the sensor array length, as seen in figure 13(b) where three profiles are shown at intervals of $0.04 \mathrm{~ms}$.

For time-dependent data, an instantaneous intermittency function $I(t)$ can be defined, taking the values 0 or 1 for states identified as laminar or turbulent, 

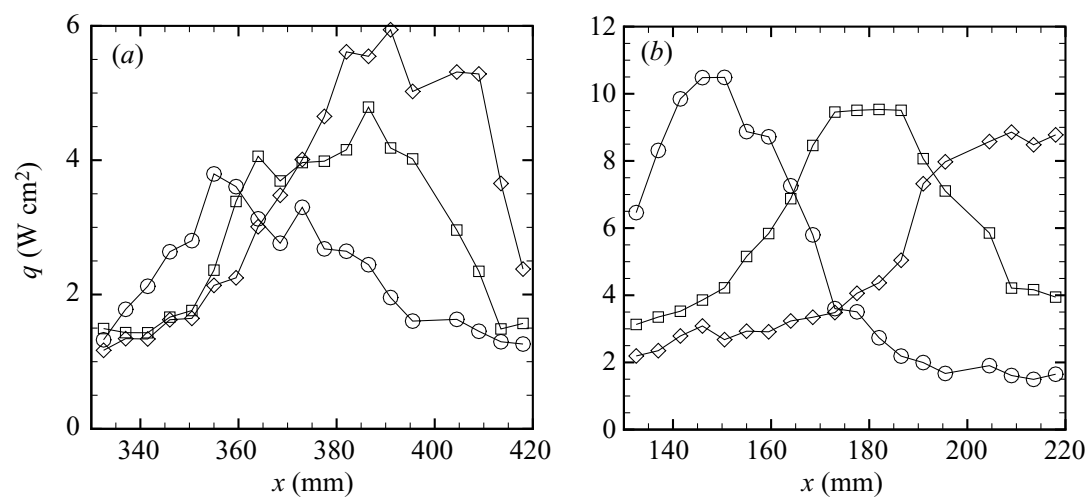

FIGURE 13. Time sequence for three axial heat transfer profiles: $(a)$ for $330 \mathrm{~mm} \leqslant x \leqslant 420 \mathrm{~mm}$, spaced at time intervals of $0.024 \mathrm{~ms} . \bigcirc, \Delta t=0 \mathrm{~ms} ; \square, \Delta t=0.024 \mathrm{~ms} ; \diamond, \Delta t=0.048 \mathrm{~ms} ;(b)$ for $130 \mathrm{~mm} \leqslant x \leqslant 220 \mathrm{~mm}$, spaced at time intervals of $0.04 \mathrm{~ms}$. $\bigcirc, \Delta t=0 \mathrm{~ms} ; \square, \Delta t=0.04 \mathrm{~ms}$; $\diamond, \Delta t=0.08 \mathrm{~ms}$.

respectively. For a sufficiently long stationary data sample, an average intermittency, $\gamma=(1 / T) \int_{0}^{T} I(t) \mathrm{d} t$, can be defined. This represents the fraction of time spent in the turbulent state. The problem, of course, is that there are no obvious absolute criteria that could be applied to the heat transfer time-histories to differentiate between turbulent and non-turbulent states. A simple threshold condition has been used, therefore, set here as a level of 1.6 times the local CFD laminar prediction. If this threshold is exceeded, the data are treated as turbulent, with intermittency $I(t)$ of $100 \%$; otherwise, the intermittency function is set to zero. Although this detection threshold might seem rather high, it was determined after some experimentation, mainly to prevent the 'noise' (electronic and, potentially, unsteady laminar) in the laminar part of the signals from triggering a false detection. In any event, it is by no means clear whether or not heating rates just above the predicted CFD steady laminar level will be turbulent, since the unsteady laminar contribution in the vicinity of a spot might be significant. This is particularly so in the base, or calm region, of a spot. The heat transfer increase at the front of spots appears to be sufficiently rapid that a precise threshold level is probably not too important in determining quite accurate positions for the front. In the wake region behind a spot, however, the heat transfer rate decays in a relatively slow manner, so that variations in threshold level would have a larger effect in determining the spot trailing-edge position. The nature of the wake region, with its gradual transition from turbulent to laminar flow, means that a definition of the spot base (certainly one based upon a surface measurement of heat transfer) is unlikely to be discriminated precisely by a simple threshold level, anyway. Figure 14 shows an example of the resultant intermittency function $I(t)$ for a short window in the distribution of figure $12(a)$.

\subsection{Spot convection speeds}

The selected sample stations in figure 12 demonstrated the streamwise convection of turbulent events, but the convection and streamwise growth of turbulent spots can be seen more easily using contour plots, in the $(x, t)$-plane, for surface heat transfer or intermittency. Figure 15 presents data from the same test used for figure 12, but based on the full array of heat transfer gauges. The $1.2 \mathrm{~ms}$ time window used for figure 15 means that the events D and A of figure 12 are captured here. The heat transfer data of figure $15(a)$ are normalized by the local CFD predictions of laminar heating rates, 


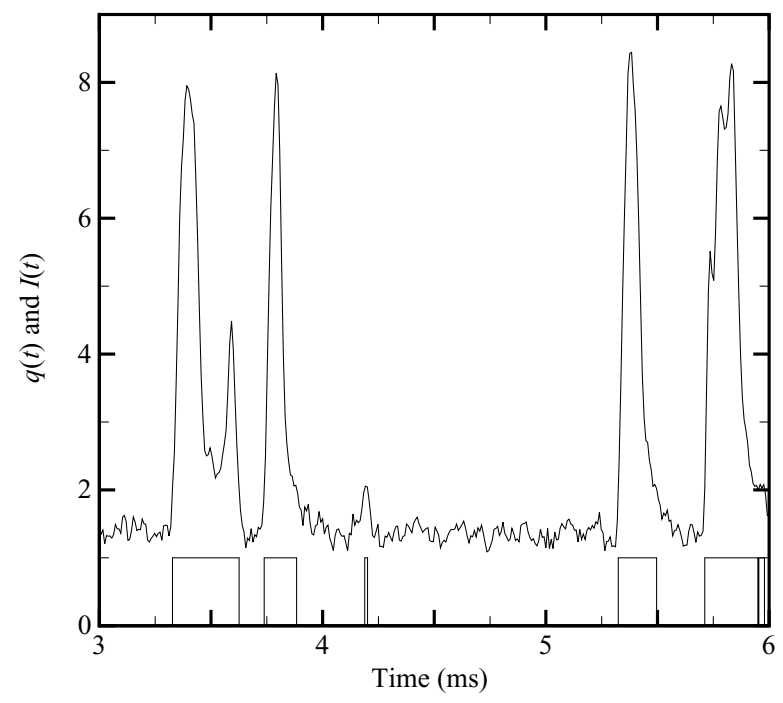

Figure 14. Comparison between heat transfer history $q(t)\left(\mathrm{W} \mathrm{cm}^{-2}\right)$ and intermittency function $I(t)$, using a threshold criterion of 1.6 times the local laminar CFD prediction. These data correpond to a segment from figure $12(a)$.

(a)

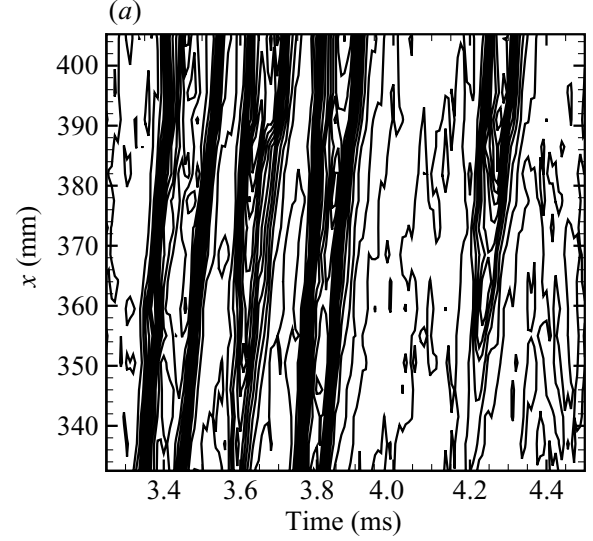

(b)

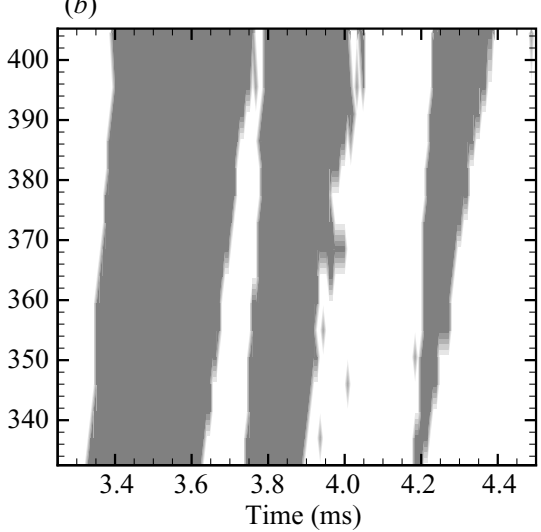

Figure 15. Presentation in the $(x, t)$-plane for the full data set corresponding to figure 12 . (a) Contours of heat transfer $q(x) / q_{L}(x)$ in intervals of $40 \%$. (b) Intermittency $I(t)$, with white corresponding to $0 \%$ intermittency (laminar flow) and dark corresponding to $100 \%$ intermittency (turbulent flow).

otherwise the effect of the streamwise reduction in laminar heat transfer levels over the sample chordwise length would be to distort slightly the perceived spreading of the spots in the $(x, t)$-plane. The slope of these contours in the $(x, t)$-plane provides an estimate of spot propagation speeds, but a more detailed evaluation requires an assessment of the trajectories of the turbulent/non-turbulent interface to be made for the 'fronts' and 'backs' of a large number of spots. An ensemble of such data is presented in figure 16 for convection over the interval $332.5 \mathrm{~mm} \leqslant x \leqslant 418 \mathrm{~mm}$ (corresponding to the data set of figure 10) and in figure 17 for convection in the range $445 \mathrm{~mm} \leqslant x \leqslant 520 \mathrm{~mm}$ (corresponding to the data set of figure 11). In these figures, distance and time are both referenced to zero at the upstream sensor of the gauge array. There is a considerable data spread in the figures. First, the average convection 

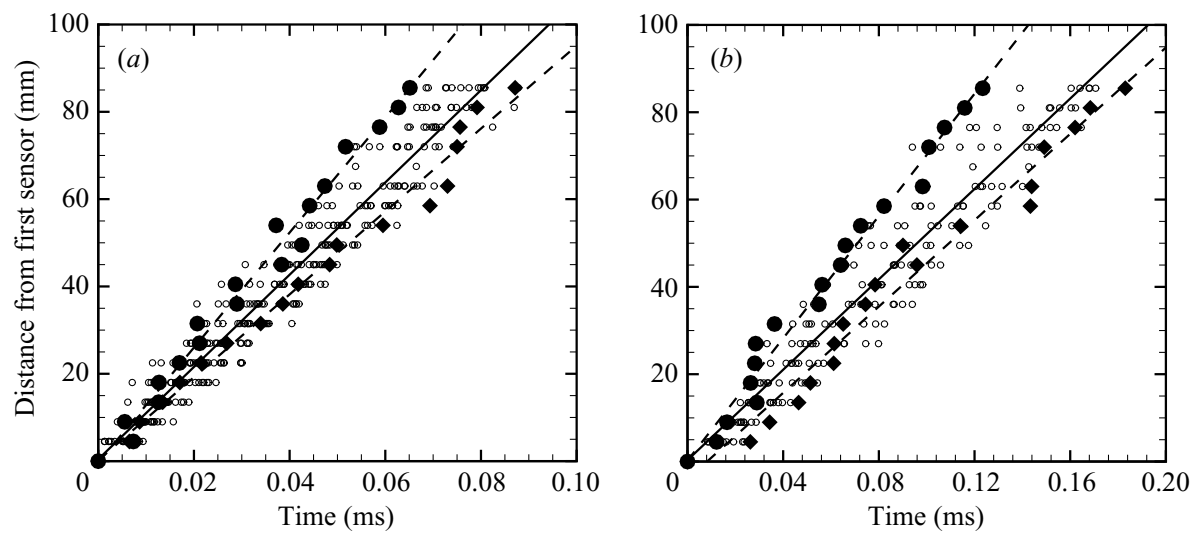

FIGURE 16. Ensemble of propagation data for $(a)$ spot fronts and $(b)$ spot backs, over the interval $332.5 \mathrm{~mm} \leqslant x \leqslant 418 \mathrm{~mm}$. The solid symbols highlight the data for the fastest and slowest propagation speeds.
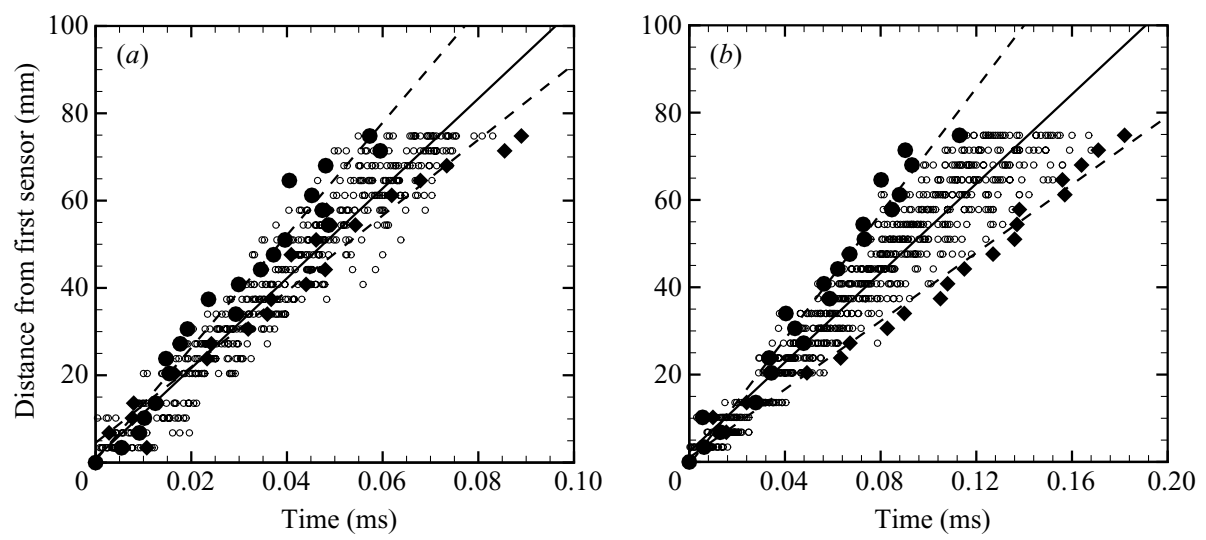

Figure 17. As figure 16, but over the interval $445 \mathrm{~mm} \leqslant x \leqslant 520 \mathrm{~mm}$.

speeds for fronts and backs, $\bar{U}_{f}$ and $\bar{U}_{b}$, can be estimated, taking a least-squares linear fit through all data points, as shown by the solid line in the figures. It is clear that values are virtually the same for the two sensor array locations, giving averaged values between the positions as $\bar{U}_{f}=1050 \mathrm{~m} \mathrm{~s}^{-1}$ and $\bar{U}_{b}=520 \mathrm{~m} \mathrm{~s}^{-1}$ or, alternatively, $\bar{U}_{f}=0.81 U_{e}$ and $\bar{U}_{b}=0.40 U_{e}$. Here, $U_{e}$, the boundary-layer edge velocity as predicted by the laminar CFD, has been averaged over the streamwise extent of the sensor array (table 2 shows that actual variations are very small indeed). The scatter in the basic data of figures 16 and 17 arises in part from random experimental errors and run-to-run variations. There are also systematic effects as shown in figures 16 and 17 which highlight the individual 'fastest' and 'slowest' trajectories for the front and back data for each data set. This almost certainly occurs because, as already noted, there is no obvious way to differentiate between signal detection on the spot (streamwise) axis and detection off the centreline. Only in the special case of a linearly growing spot, with a constant apex speed and a constant-sweep leading edge, will measurements, at a fixed transverse offset from the spot axial centreline, produce a convection speed independent of transverse position. A linearly growing spot with a curved leading edge will show a dependence of the inferred convection speed on the 

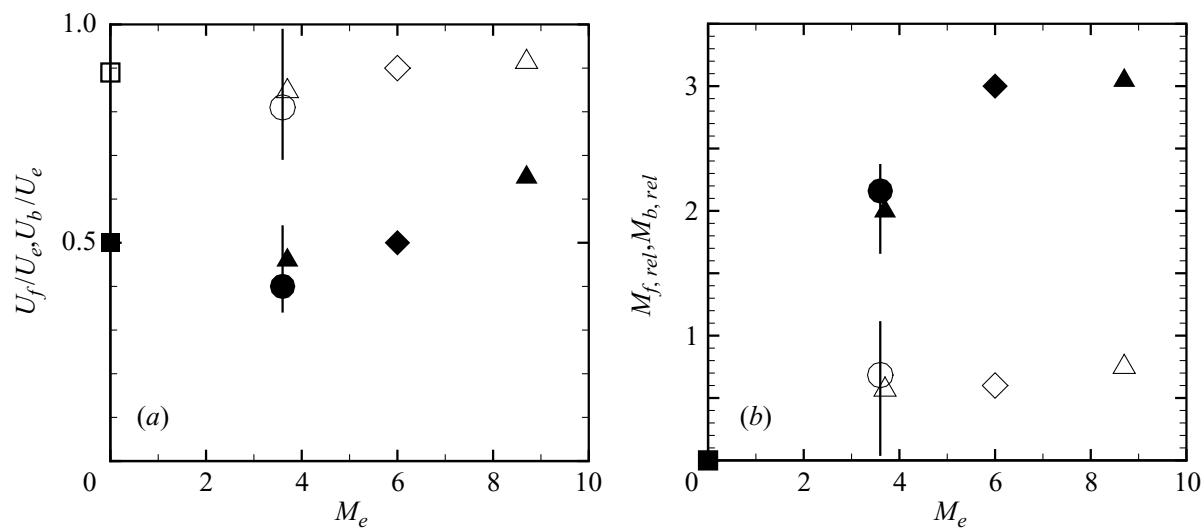

FigurE 18. Collection of various data for front and back speeds. $\square$, Wygnanski et al. (1976); $\bigcirc$, present; $\triangle$, James (1958); $\diamond$, Mee (2002). Solid and open symbols represent back and front speeds, respectively. The vertical bar on the present data denotes the extreme range of values found experimentally.

transverse position of the sensor array relative to the spot axis. Depending upon the sense of the mean front curvature, this measured speed may exceed or fall below the speed of the spot apex, and would also be affected by local corrugations in the front, seen in the later contour plots of $\S 4.3$. If space-time measurements are made over a substantial axial distance - significantly greater than the possible sensor array length here - then the average speed will tend progressively to that of the apex. Because 'front' detection appears to be quite accurate (but obviously slightly underestimating the position and hence velocity), a significant cause of the scatter has to reflect these real physical variations of inferred propagation speed across the spanwise extent of the front. The fastest front propagation speed in figure 16 and 17 is $1310 \mathrm{~m} \mathrm{~s}^{-1}$, virtually the same as the computed boundary-layer edge velocity, and the lowest front trajectory speed is approximately $0.69 U_{e}$. The scatter in figures $16(b)$ and $17(b)$, for the back trajectories, again reflects the same points as made for the fronts, but also the fact that the more gradual variation of surface heating in the base region is a possible cause of lack of sensitivity or variability in detection. This last point will be reconsidered in the next section because of its role in defining the spot planform shape. The 'maximum' back speed in figure $16(b)$ is $0.54 U_{e}$ and the lowest back speed is between $0.30 U_{e}$ and $0.38 U_{e}$ but, as already noted, it is not possible to judge whether the latter is a turbulent/non-turbulent interface, or is already in the calm/base region. Figure 18 presents data, from various studies, for the front and back convection speeds (expressed as a fraction of the edge velocity) as well as the relative Mach number between the boundary-layer edge flow and the spot front or back, for example $M_{f, r e l}=\left(U_{e}-U_{f}\right) / a_{e}$, where $a_{e}$ is the speed of sound at the boundary-layer edge. In this figure, the data for Wygnanski et al. (1976) and James (1958) are direct observations of the apex and base (i.e. rearmost position) speeds. The data of Mee (2002) is likely to include an element of averaging across the front, similar to the above observations. The current front speed data point in the figure is the average result, but a 'scatter bar' is included to denote the extreme speeds determined from figures $16(a, b)$ and $17(a, b)$.

An estimate of the mean spot convection speeds can also be made from the spacetime correlation data between pairs of sensors. Figure 19 shows the time-lagged 


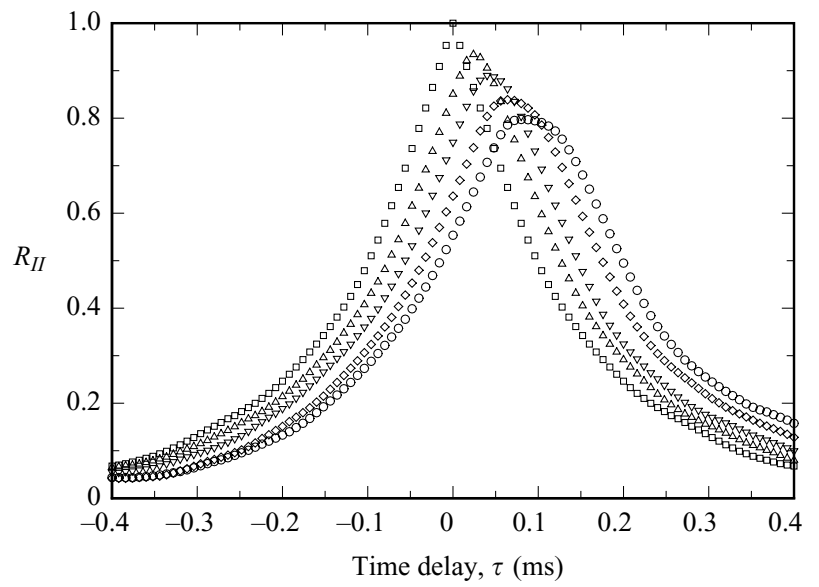

FIGURE 19. Typical space-time correlation of intermittency between sensor pairs using the data set from figure 11. Spacing $\Delta x: \square, 0.0 \mathrm{~mm} ; \Delta, 20.5 \mathrm{~mm} ; \nabla, 37.4 \mathrm{~mm} ; \diamond, 57.8 \mathrm{~mm} ; \bigcirc$, $74.8 \mathrm{~mm}$.

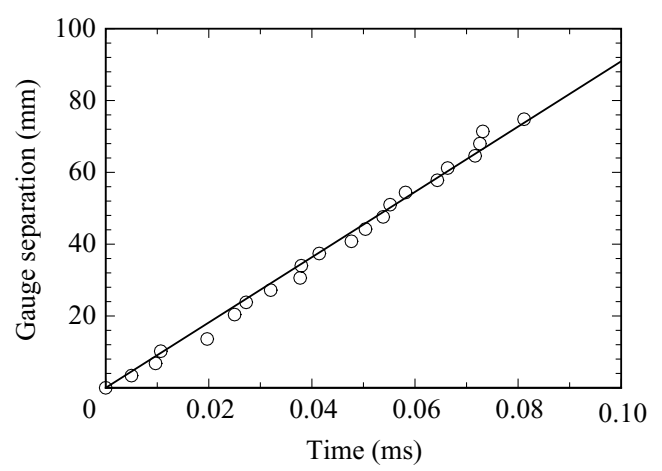

FIGURE 20. Composite $(x, t)$ diagram for all gauge pairs.

correlation for intermittency, using two sensors, one fixed at $x=445 \mathrm{~mm}$ and the other variable in position up to $520 \mathrm{~mm}$, using the full data set employed for figure 11. The mean convection time between the two sensors is taken as the time delay to the correlation maximum, plotted in figure 20 for the full range of sensor spacings, and providing a mean convection speed of approximately $905 \mathrm{~m} \mathrm{~s}^{-1}$ or $0.69 U_{e}$. The intermittency function has been selected for the correlation as being the most direct measure of spot propagation, although correlations based upon the full heat transfer history are little different and provide a mean convection speed of $0.66 U_{e}$. These assessments are somewhat larger than the arithmetic average of $\bar{U}_{f}$ and $\bar{U}_{b}\left(U_{C} \approx 0.6 U_{e}\right)$, probably in part because the assessments of $\bar{U}_{f}$ and $\bar{U}_{b}$ used a selected database of identifiable individual spots, whereas the cross-correlation is a full ensemble of data. The data for $U_{C}$ are compared in table 3 with other experimental sources for a range of Mach numbers.

\subsection{Spot planform geometry}

In the same way that the time histories and spatial distributions of figures 12 and 13 provide data on the axial distributions, the simultaneous heat transfer histories from the circumferential array of sensors provide data on the transverse scale and 


\begin{tabular}{llcc}
\multicolumn{1}{c}{ Study } & Spot type & $M_{e}$ & $U_{c} / U_{e}$ \\
Schubauer \& Klebanoff & Artificial & 0.03 & 0.69 \\
Wygnanski et al. & Artificial & 0.02 & 0.70 \\
Cantwell et al. & Artificial & $\approx 0$ & 0.72 \\
Coles \& Savas & Artificial & 0.03 & 0.76 \\
Matsui & Artificial & $\approx 0$ & 0.68 \\
Gutmark \& Blackwelder & Artificial & 0.01 & 0.73 \\
Sankaran et al. & Artificial & 0.01 & 0.71 \\
Clark et al. & Natural & 0.24 & 0.71 \\
Clark et al. & Natural & 0.55 & 0.69 \\
Clark et al. & Natural & 1.32 & 0.70 \\
Clark et al. & Natural & 1.86 & 0.68 \\
James & Natural & 3.7 & 0.67 \\
James & Natural & 8.7 & 0.78 \\
Mee & Natural & 6.0 & $\approx 0.7$ \\
Present & Natural & $3.2-3.7$ & $0.6-0.69$
\end{tabular}

TABLE 3. Comparison of convection speed for current and previous studies at various values of Mach number external to the boundary layer, $M_{e}$.

structure of the turbulent spots. This is best visualized by a contour plot of surface heat transfer in the $(t, z)$-plane. Four typical results, at each of the three positions $x=213 \mathrm{~mm}, x=279 \mathrm{~mm}$ and $x=347 \mathrm{~mm}$, are shown in figures 21,22 and 23 , respectively. These cases have been selected because they are contained, as far as is possible for the rearmost station, within the circumferential span of the set of 18 gauges. Many spots were only partially contained within the sensor array, or were in the process of amalgamating with other spots, and both such types of event have generally been discarded here. The spanwise scale, $z$, is the transverse wetted distance around the circumference and is normalized by the CFD prediction for the local laminar displacement thickness, $\delta_{1, L}$. The zero time origin in each case has been fixed as close as possible to the instant when the maximum spot width coincides with the measurement station and this adjusted time is normalized as $-t U_{c} / \delta_{1, L}$, where $U_{c}=0.5\left(\bar{U}_{f}+\bar{U}_{b}\right) \approx 0.6 U_{e}$ is the convection velocity defined earlier. If spots were to convect as frozen structures such a normalization would convert time-histories to (nearly) the correct spatial shape, but clearly this is not the case here. The earlier convection-speed measurements show that the apex speed must be about twice the base velocity. A spot can therefore be expected effectively to double in length (and probably in width therefore) in the time interval from the spot apex first reaching a sensor location to the spot trailing edge just passing it. The true physical planform scale and shape of a spot, in the $(x, z)$-plane, can only be inferred from the $(t, z)$ data if assumptions are made about the spatial convection speed characteristics. It also requires the best possible definition of the planform shape in the $(t, z)$-plane. The contour plots $(t, z)$ of figures 21 to 23 suggest a highly swept front and the impression of a slightly re-entrant base, but the actual $(t, z)$ shape depends upon the simple criterion $\left(q_{s p o t} \geqslant 1.6 q_{L}\right)$ used for detection of the spot interface. Figure 24 , as an example, takes the first two planforms of figure 22, presenting contour plots for the range $1.6 \leqslant q / q_{L} \leqslant 2.6$. It would appear from these that the apex and the sides of the spot (to somewhere downstream of the maximum width position) are quite well defined and that the exact interface position is not too sensitive to the threshold level chosen. Estimates of spot width - and hence its growth with streamwise distance - will 

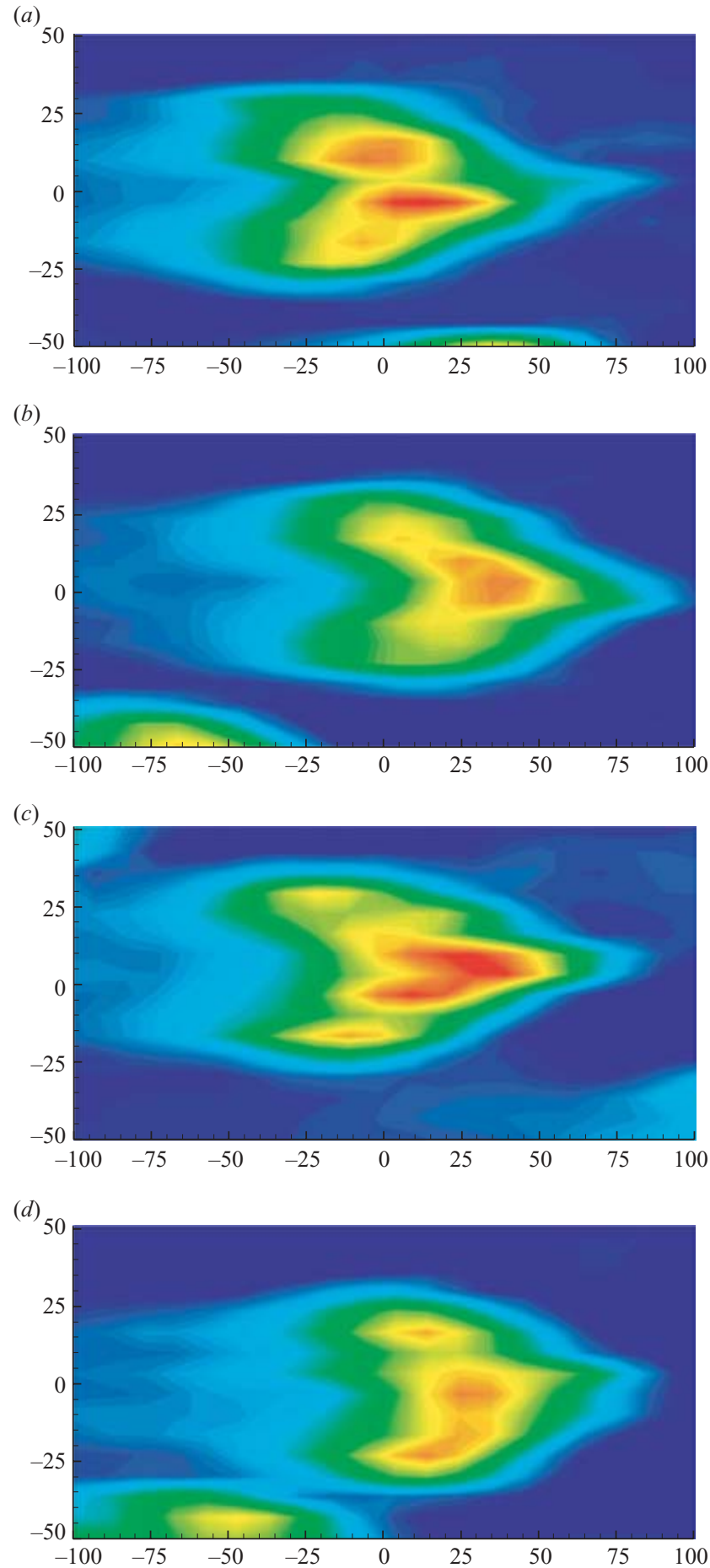

FIGURE 21. Heat transfer contours in the $(t, z)$-plane for detection of individual spots using the circumferential array of gauges, at $x=213 \mathrm{~mm}$. The ordinate is $z / \delta_{1, L}$, the transverse wetted distance, $z$, normalized by the local laminar displacement thickness, $\delta_{1, L}$ (computed as $0.607 \mathrm{~mm}$ from CFD). The abscissa is $-\tilde{t} U_{C} / \delta_{1, L}$, where $\tilde{t}$ is time and $U_{C}$ is the mean convection speed. 


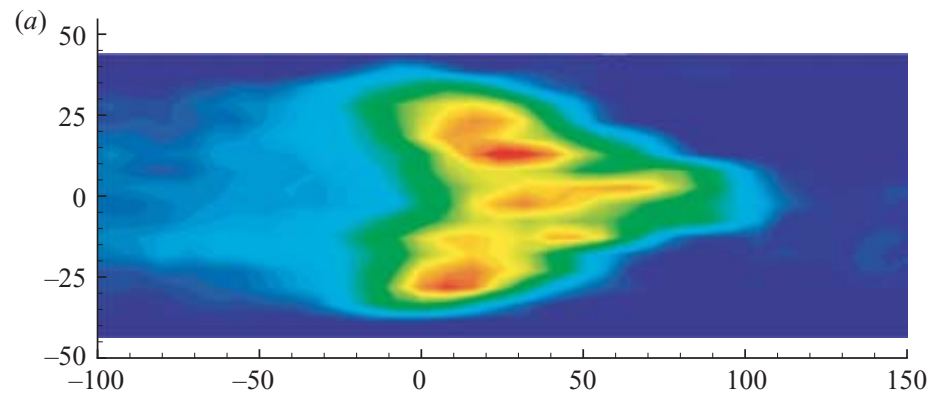

(b)

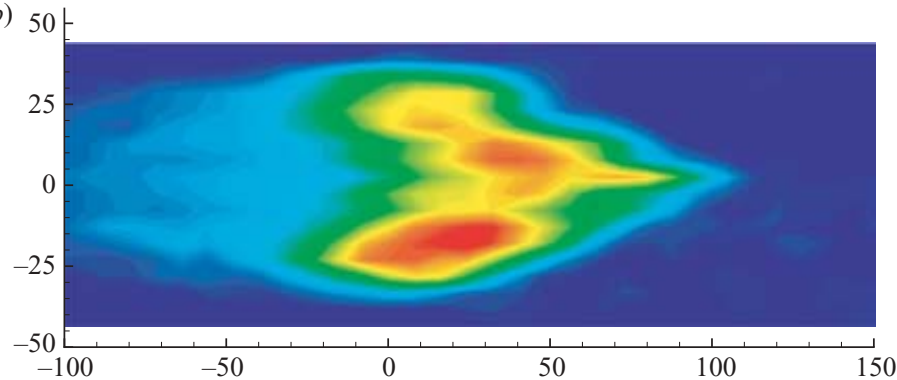

(c)

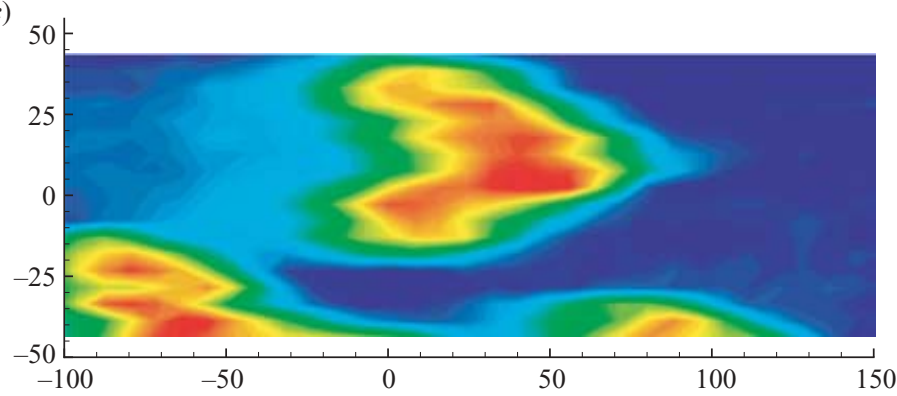

(d)

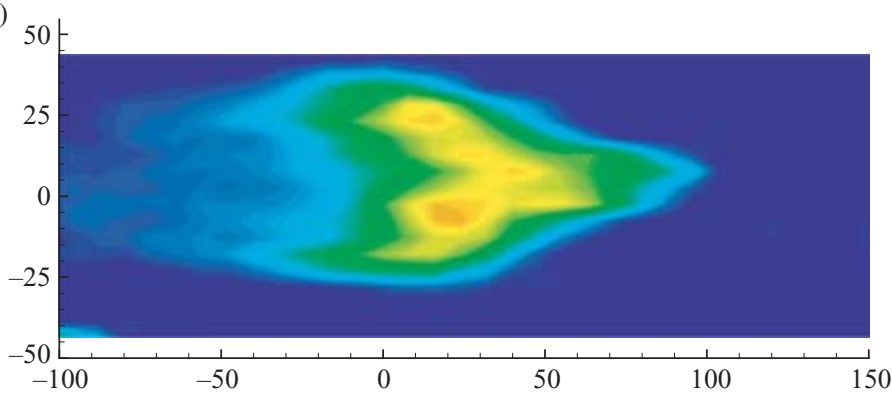

Figure 22. As for figure 21, with $x=279 \mathrm{~mm}, \delta_{1, L}=0.78 \mathrm{~mm}$.

therefore be fairly accurate. Recalling that spots grow continuously with distance, it is important to note that the spot width, therefore, is defined at the instant when the maximum width location of the spot coincides with the spanwise sensor array. Figure 24 shows that the base region of the spot is more sensitive to the threshold level; it would be debatable whether to reduce the threshold, which would elongate even further the base region and reduce the apparent base convection speed, or to increase it. 

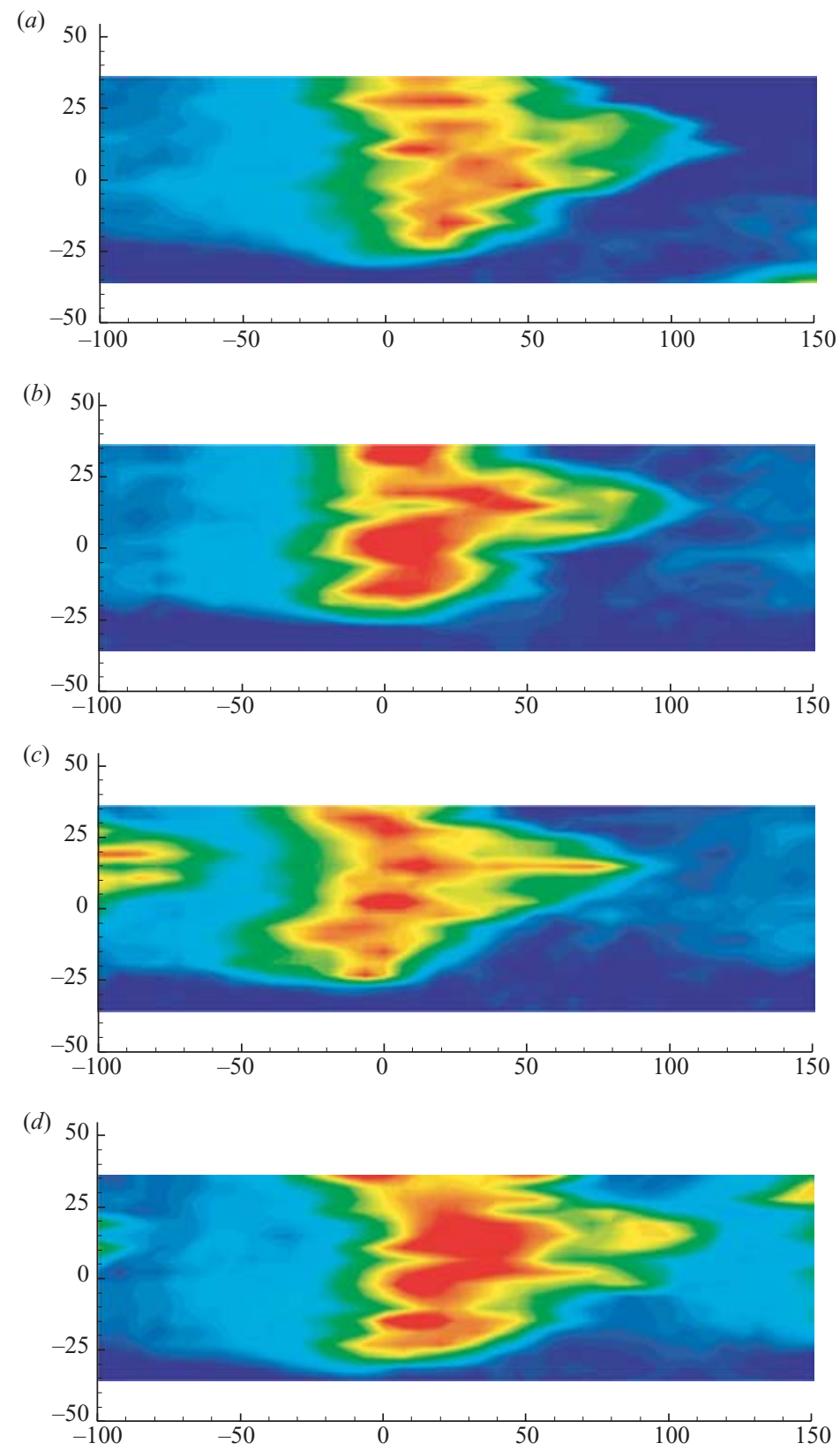

FIGURE 23. As for figure 21, with $x=347 \mathrm{~mm}, \delta_{1, L}=0.947 \mathrm{~mm}$.

Estimating the physical length of spots is more difficult than determining the width. Assuming that the overall planform shape of the spot grows in a self-similar manner with time, with constant apex and back convection speeds, the spatial scales can be estimated using figure 25. Figure $25(a)$ shows a spot at an arbitrary time $t_{0}$ after its inception, when its maximum width position is located at $x_{0}$. The $(x, t)$ plot of figure $25(b)$ shows the spot apex and back trajectories, given by $\mathrm{d} x / \mathrm{d} t=U_{f}, U_{b}$, respectively. The horizontal distance $x_{f}-x_{b}$ represents the streamwise extent of the 

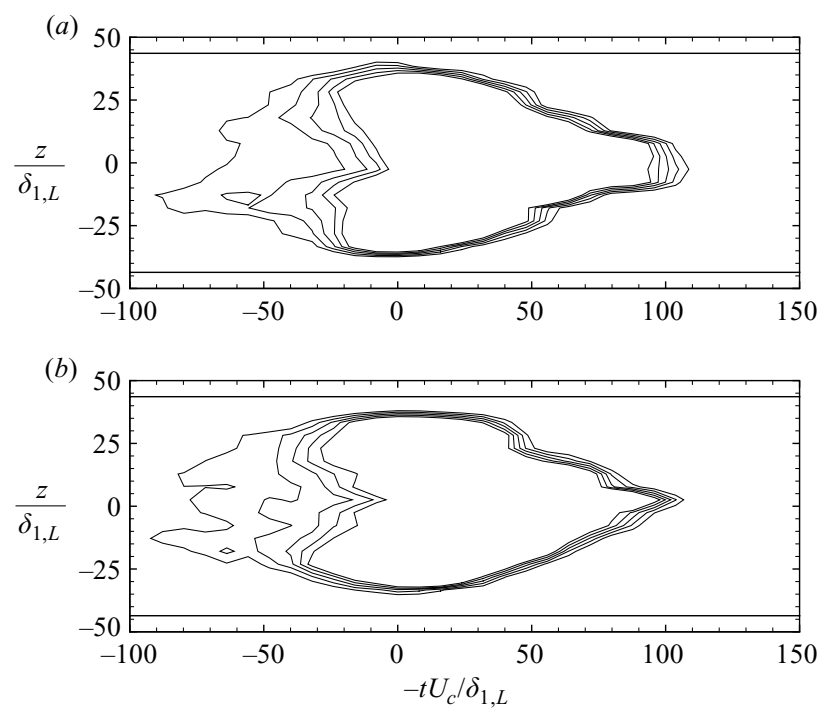

FIGURE 24. Data from figure 22 presented as contours over the interval $1.6 \leqslant q / q_{L} \leqslant 2.6$ in steps of 0.25 .

(a)
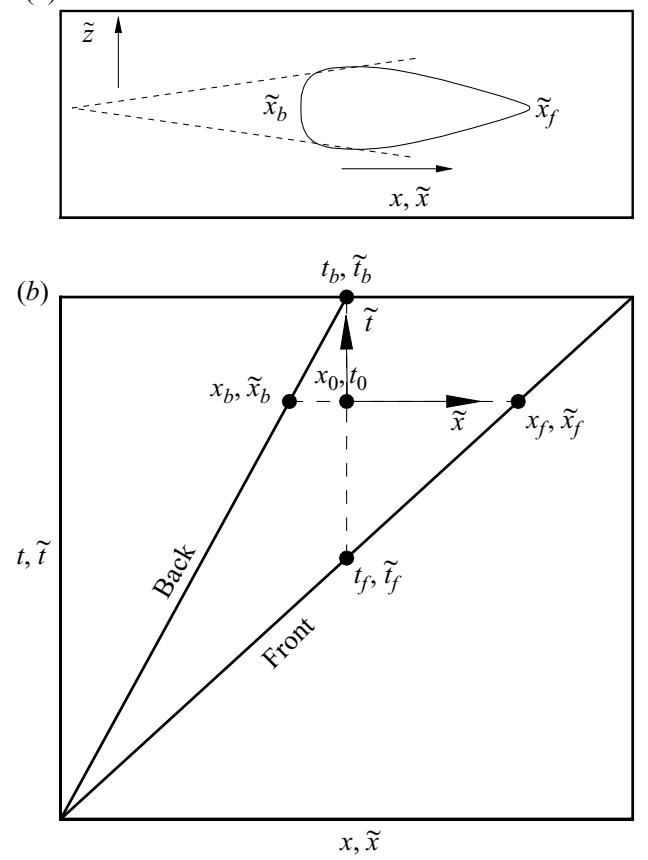

FIgURE 25. Time-distance diagram for estimation of spot length. $X_{0}$ is a spot development length, or virtual development length, assuming linear growth rates.

spot at the instant $t_{0}$, between time $t_{f}$ when the spot apex first reaches the measuring station and $t_{b}$ when the back just reaches it. It is convenient to reference time to zero at $t_{0}$ so that $\tilde{t}$ and $\tilde{x}$ are the perturbations from $t_{0}$ and $x_{0}$, respectively. This is the form that was used in figures 21 to 23 . It then follows that the two limiting spot 


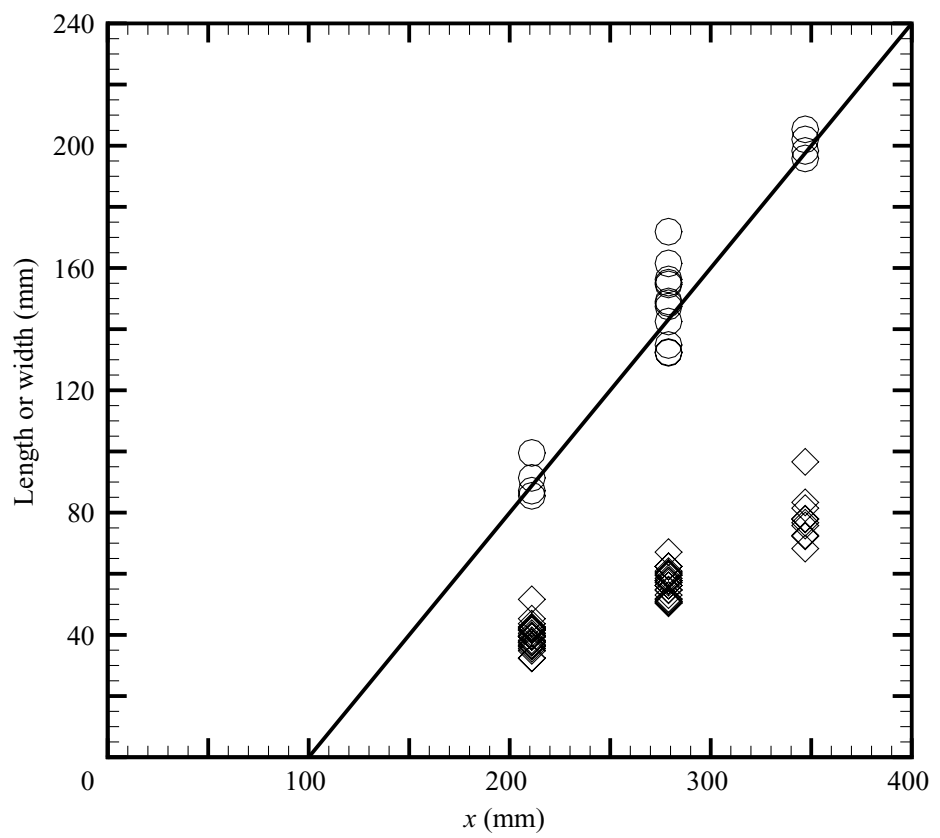

FIGURE 26. Variation of $\bigcirc$, spot length $l_{s, 0}$, according to equation (4.3) and $\diamond$, maximum width, with axial distance. The trend line shows the rate of growth prediction for $l_{s, 0}$ according to equation (4.7).

lengths, $l_{s, f}$ and $l_{s, b}$, corresponding to the cases where the front or back, respectively, reach the sensor location, and the spot length $l_{s, 0}$ at time $t_{0}$ when the maximum width position coincides with the sensor array location, are given by

$$
\begin{aligned}
l_{s, f} & \approx\left(\tilde{t}_{b}-\tilde{t}_{f}\right) U_{b}, \\
l_{s, b} & \approx\left(\tilde{t}_{b}-\tilde{t}_{f}\right) U_{f}, \\
l_{s, 0} & \approx \tilde{t}_{b} U_{b}-\tilde{t}_{f} U_{f} .
\end{aligned}
$$

The arrival times $\tilde{t}_{f}, \tilde{t}_{b}$ for the apex and back detection are determined for each individual spot from plots such as figure 24. Strictly, the values for $U_{b}$ and $U_{f}$ in (4.1) to (4.3) should be the apex and base (centreline) speeds. However, it makes only a small difference if the mean values $\bar{U}_{f}$ and $\bar{U}_{b}$, that is the average values across the whole front and base of the spot, respectively, are used instead. Thus, for the whole ensemble of spot data, the average values for $l_{s, 0}$ are $91 \mathrm{~mm}, 149 \mathrm{~mm}$ and $200 \mathrm{~mm}$, respectively, for the locations $x=213 \mathrm{~mm}, 279 \mathrm{~mm}$ and $347 \mathrm{~mm}$ if $\bar{U}_{f}$ and $\bar{U}_{b}$ are used. If a maximum apex speed of $0.9 U_{e}$ were assumed, together with a minimum base speed of $0.35 U_{e}$, which encompasses the main scatter range in figure 18 , then these estimates for the spot length would only be increased by $7 \%$. Figure 26 presents data for individually predicted spot lengths $l_{s, 0}$ and measured spot widths. The accumulated effect of errors - determining $U_{f}, U_{b}$ and $\tilde{t}_{f}, \tilde{t}_{b}$, together with the sensor data sampling resolution - is at least $\pm 10 \%$. Accepting this, it still appears from figure 26 that, at any $x$-position, spot scales fall within a narrow band. This implies that the spot inception region is also narrowly constrained in terms of streamwise distance and that earlier comments on figure 12, concerning the origin of 'new spikes' in the heat transfer time-histories, indicate that they probably therefore 
arise from the 'edges' of spots sweeping over the sensor rather than being the origin of new disturbances.

Projecting the data of figure 26 back to a virtual origin would indicate the range $60 \mathrm{~mm} \leqslant x \leqslant 110 \mathrm{~mm}$ for spot inception, which corresponds geometrically to the blending section of the body in figure 3. This is a region of rapid decrease with distance of the magnitude of the favourable pressure gradient (see figure 7) and hence presumably of loss of a stabilizing influence. Whether the inception position is associated specifically with the discontinuities in surface curvature, between the blending section and the spherical nose and cylindrical afterbody, respectively, is not known. From the viewpoint of the surface pressure a curvature-blended shape would redistribute the surface pressure gradient profile only slightly, so that it is doubtful if such a change would provide any significant shift in inception position. The only other relevant work is an experiment on blunted cones by Stetson (1988), who compared transition onset data at Mach 5.9 for a sphere-cone configuration (with surface streamwise-curvature discontinuity therefore) and a curvature-blended sphere-cone. Unlike the present case, the sphere-cone produces an overexpansion (relative to the asymptotic cone surface pressure) downstream of the curvature discontinuity and hence the appearance eventually of an adverse pressure gradient. With removal of this, by curvature blending, Stetson found little effect on the results.

Based on figure 25, further alternative expressions for spot lengths can be made, that is

$$
\begin{gathered}
l_{s, f} \approx x_{0}\left(1-\frac{U_{b}}{U_{f}}\right), \\
l_{s, b} \approx x_{0}\left(\frac{U_{f}}{U_{b}}-1\right), \\
l_{s, 0} \approx x_{0}\left(\frac{U_{f}}{U_{\alpha}}-\frac{U_{b}}{U_{\alpha}}\right) .
\end{gathered}
$$

Here, $U_{\alpha}=U_{b}+\alpha\left(U_{f}-U_{b}\right)$ is the convection speed associated with the maximum width position of the spot and $\alpha$ is the fractional distance from the base to this maximum width position. Equating equations (4.3) and (4.6) then establishes $x_{0}$, the development distance of the spot from the virtual inception position. Averaged over all data, the value of $\alpha$ was approximately 0.25 , so that the rate of growth of spot length, with distance, is given as

$$
\frac{\mathrm{d} l_{s, 0}}{\mathrm{~d} x} \approx 0.8
$$

The values of $x_{0}$ for each spot measurement station independently provided a virtual inception position $x \approx 100 \mathrm{~mm}$, for the above assumption that $U_{f} \approx \bar{U}_{f} \approx 0.81 U_{e}$ and $U_{b} \approx \bar{U}_{b} \approx 0.40 U_{e}$ and the corresponding trend line from equation (4.7) is shown in figure 26. If the upper and lower estimates are taken for $U_{f} \approx 0.9 U_{e}$ and $U_{b} \approx 0.35 U_{e}$, respectively, which extends the spot length prediction by $7 \%$, the virtual spot inception position would only be some $20 \mathrm{~mm}$ earlier.

The modelling of figure 25 and equations (4.1) to (4.6) can be extended to approximate the complete planform shape. Once a value for $x_{0}$ has been determined (taking, here, $x=100 \mathrm{~mm}$ for spot inception), a self-similar growth of the spot planform would scale the streamwise and spanwise scales (noting that $x_{0}=U_{\alpha} t_{0}$ ) 

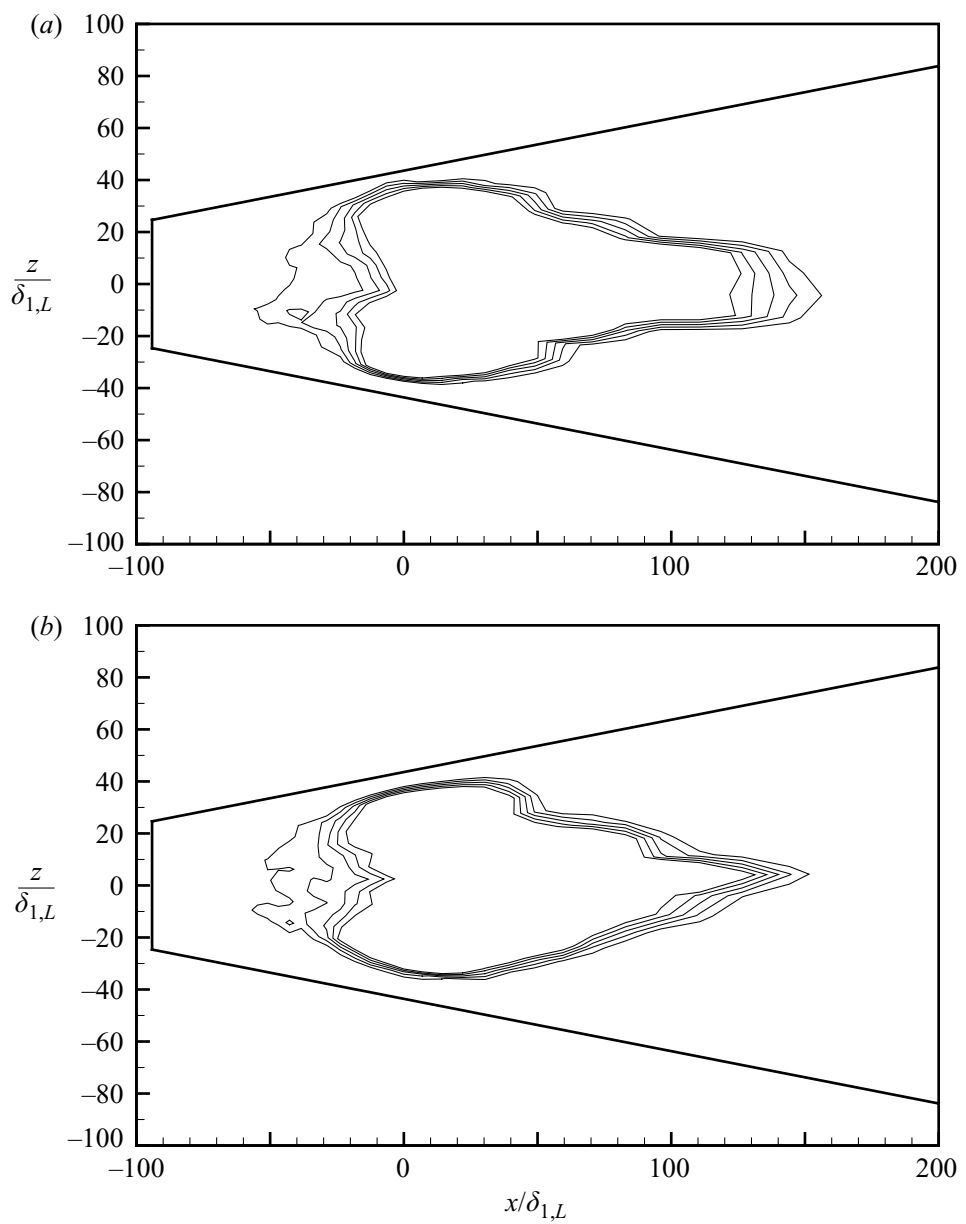

FIGURE 27. Data from figure 24, scaled according to equations (4.8) and (4.9), presented as contours over the interval $1.6 \leqslant q / q_{L} \leqslant 2.6$.

Semi-spreading angle

Length/width ratio

Semi-apex angle

Maximum width position/spot length $(\alpha)$

$$
\begin{gathered}
6.75^{\circ} \pm 1.0^{\circ} \\
2.5 \pm 0.25 \\
17^{\circ} \pm 3^{\circ} \\
0.25 \pm 0.05
\end{gathered}
$$

TABLE 4. Average properties for spots.

from the $(t, z)$ histories, as

$$
\begin{aligned}
& \frac{\tilde{x}}{x_{0}}=-\frac{\tilde{t} / t_{0}}{1+\tilde{t} / t_{0}}, \\
& \tilde{z}(\tilde{x})=\frac{z}{1+\tilde{t} / t_{0}} .
\end{aligned}
$$

Figure 27 shows the resultant scaling of the two sample $(t, z)$ spot shapes of figure 24. Table 4 provides averaged properties for all spots scaled according to equations (4.8) and (4.9). The half-angle spreading rate, taken from the data of 
figure 26, is high compared with the trend presented by Fischer (1972 b) (see figure 2), which suggested spreading rates of $3.5^{\circ}$ to $5.5^{\circ}$ at this edge Mach number. The difference compared to Fischer's data is unlikely to be significant, given the difficulty generally of defining a precise edge and the fact that in figure 2 the growth rates vary sensitively with Mach number below Mach 4.0. For some of James' data, at Mach 3.9, he gives semi-spreading angles up to $11^{\circ}$, significantly larger than any shown by Fischer. His spot leading-edge angles (i.e. semi-apex angle) range from $10^{\circ}$ to $18.5^{\circ}$ and are therefore consistent with the averaged values for the present data in table 4 .

Equations (4.8) and (4.9) produce a plausible scaling for the spot planform shape, but there is no clear mechanism to map the thermal planform contours from the $(\tilde{t}, z)$-plane to the $(\tilde{x}, \tilde{z})$-plane. Figure 28 presents thermal planforms for four scaled spots, two from the sequence of figure 22 and two from figure 23 , by simply assuming that $q / q_{L}$ maps unaltered between equivalent positions. The spot planforms are similar to those found in lower-speed studies with a triangular front, a blunt or slightly recessed base, and a 'wake' region. A typical axial distribution along the centreline of a spot is shown in figure 29 for the second spot in figure 22(b) (or figure 28b). Figure 29(a) shows the original time-history and figure 29(b) presents it after transformation to the physical scale $\tilde{x}$. The effect of the $2: 1$ difference in convection speed between fronts and backs becomes evident in the change of relative steepness and this feature is also evident in the instantaneous axial profile measurements of figure 13. It still remains that a threshold heat transfer level of 1.6 times the laminar CFD prediction identifies the front quite accurately. By contrast, the most obvious feature in the base distribution is the reduction in heat transfer gradient, when the mean level is about 2.1 times the laminar value, significantly above the threshold actually used. If this were the true identifier of the turbulent/nonturbulent interface, then the typical base convection speed would be of order $0.48 U_{e}$. The region in figure $29(a, b)$ in front of the spot, $-\bar{t} U_{c} / \delta_{1, L} \geqslant 100$ or $\bar{x} / \delta_{1, L} \geqslant 150$, indicates a slight depression in heating levels, probably because the spot is convecting in the wake region of a preceeding spot. Figure $29(c)$ shows the transverse distribution through the spot at the position of maximum width $(\tilde{x}=0)$.

A feature of the planform contours of figures 21 to 23 and figure 28 is the apparent 'cellular' structure. Spatial resolution of the measurements is coarse (see $\$ 2.2$ ), but still sufficient to show that this internal structure is a real effect, and not the result of any gauge-to-gauge variation. The cell numbers increase with the increase in overall scale of the spot, seen by comparing figures 21 and 23, with typical spanwise spacings $\Delta z$ between 'cells' of 10 to 15 in $\Delta z / \delta_{1, L}$. This may connect with low-speed studies (e.g. Cantwell et al. (1978) and the schematic by Perry et al. (1981) in figure 30) which indicate an internal 'streak' or 'cellular' structure. A faint streak pattern is also visible in the 'calm' region downstream of the spot, similar to streaks shown, amongst others, by Carlson, Widnall \& Peeters (1982) for incompressible flow.

\section{Concluding remarks}

The measurements have focused entirely upon time-dependent heat transfer, both because this is an easily measured quantity of practical importance and also because it provides an excellent surface footprint for the turbulent spot. Measurements to produce instantaneous spot geometries are difficult and the present data probably provide a unique data set for hypersonic flows that gives both a measure of the overall scales, and propagation speed, of spots and has also resolved the data sufficiently well to indicate detail of an internal cell structure. The spot data show many similarities 

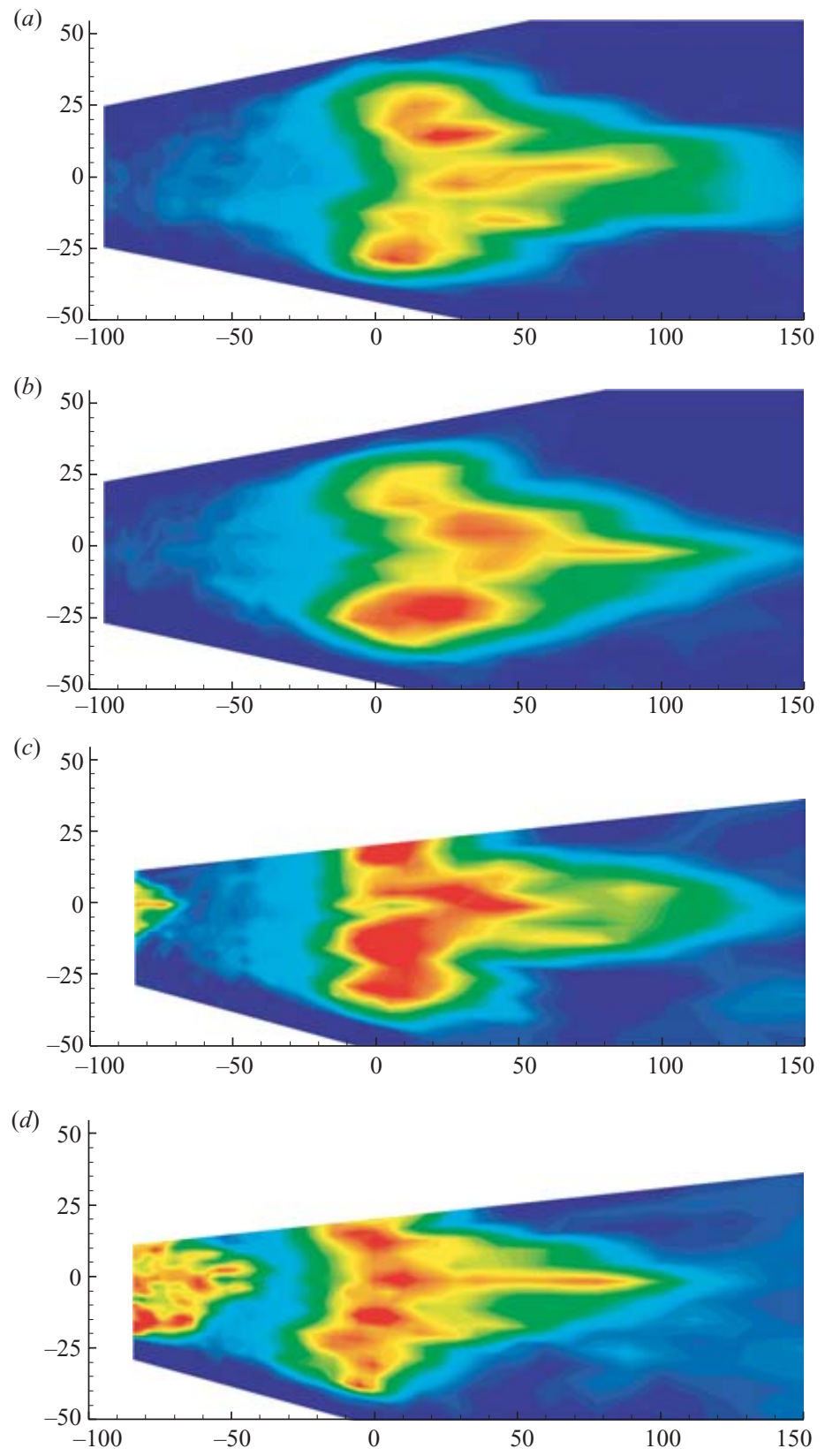

FIGURE 28. $(t, z)$ distributions scaled to spot plan forms in the $(\tilde{z}, \tilde{x})$-plane, using the scalings of equations (4.8) and (4.9). $\tilde{x}$ and $\tilde{z}$ are both normalized by the laminar boundary-layer displacement thickness $\delta_{1, L}$ as in figure 27. The top two spots are taken from figure 22 and the bottom two from figure 23 .

with low-speed studies and those obtained at lower supersonic, or transonic, speeds although spot growth rates are high compared with the data correlation of Fischer $(1972 b)$. It has not been possible to make comments on receptivity, or fully identify the spot inception zone. The spots appear to arise close to the curvature discontinuities 

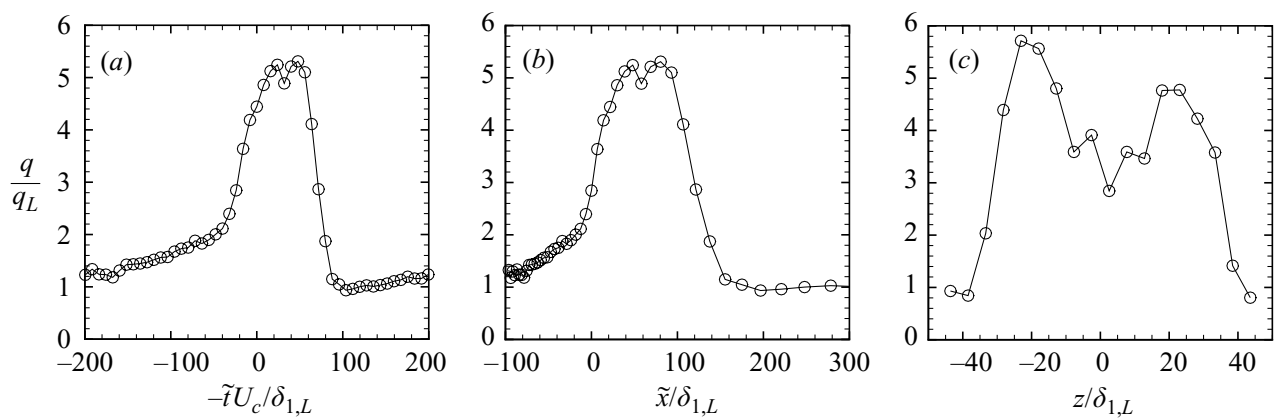

FiguRE 29. Detail for a turbulent spot from figure 22(b) based upon the sensor array at $x=279 \mathrm{~mm}$. (a) Axial distribution with time $\tilde{t}$ of heat transfer along centreline; $(b)$ axial distribution with distance $\tilde{x}$ of heat transfer along centreline; (c) transverse distribution of heat transfer at maximum thickness position. All heat transfer data are normalized by the reference laminar value.

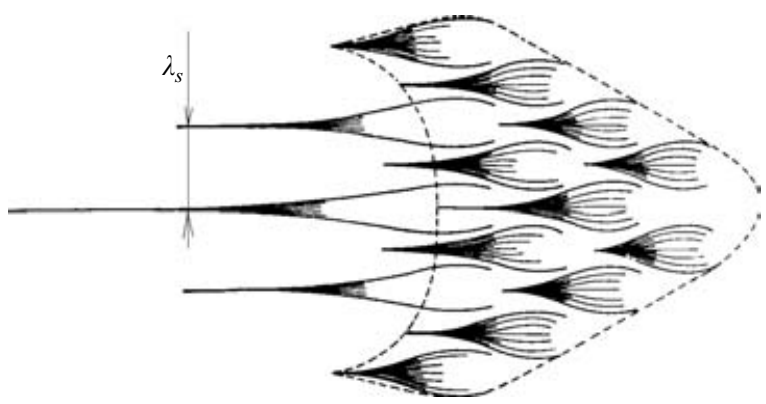

FIGURE 30. Schematic for internal structure of a turbulent spot at low speeds, taken from Perry et al. (1981).

associated with the blending zone between the spherical nose and the cylindrical afterbody, although it is suspected that it is the rapid reduction in favourable pressure gradient, rather than the curvature discontinuity, that is mainly responsible. The subsequent spot development is then in a region of continuous, weak favourable pressure gradient. The main characteristic of this region is the strong entropy-layer effect, so that although the free-stream conditions are a nominal $M_{\infty}=8.9, R e_{\infty}=$ 47.4 million per metre, the edge conditions for the bulk of the spot development are closer to $M_{e} \approx 3.5$ and $R e_{e} \approx 2.9$ million per metre. This is in the region of socalled transition reversal, induced by sufficiently large nose bluntness. In this regime, boundary-layer transition is particularly sensitive to roughness in the blunt-nose region and immediately downstream. The main spot planform data presented here have been recorded with the highly polished model state, which provides a sparse spot production rate so that individual spots, grown to large size, are monitored at large chordwise distances. In many other areas, such large stretches of intermittent flow would never be realized. At hypersonic speeds, it clearly can comprise a substantial part of the flow field and many critical interactions for hypersonic boundary layers are then potentially associated with a transitional boundary layer, rather than a fully laminar or turbulent one. 
We would like to gratefully acknowledge Mr R. Hutchins and Mr S. Johnston for their expertise in model manufacture and operation of the wind tunnel. S. G. M. would like to acknowledge the generous support of the Australian Academy of Science through the Scientific Visits to Europe Program. A. F. was supported by the Algerian Ministry of Higher Education and Scientific Research and by the Overseas Research Student Award scheme.

\section{REFERENCES}

Ben-Artzi, M. \& Falcovitz, J. 1984 A second-order Godunov-type scheme for compressible fluid dynamics. J. Comput. Phys. 55, 1-32.

Cantwell, B., Coles, D. \& Dimotakis, P. 1978 Structure and entrainment in the plane of symmetry of a turbulent spot. J. Fluid Mech. 87, 461-672.

Carlson, D. R., Widnall, S. E. \& Peeters, M. F. 1982 Transition in plane Poiseuille flow. J. Fluid Mech. 121, 487-505.

Clark, J. P., Jones, T. V. \& LagrafF, J. E. 1994 On the propagation of naturally occurring turbulent spots. J. Engng Maths 28, 1-19.

Clark, J. P., Jones, T. V. \& LagrafF, J. E. 1996 Turbulent-spot propagation in a decelerating, compressible flow. Trans. AMSE I: J. Fluids Engng 118, 612-614.

Coles, D. \& Savas, O. 1980 Interactions for regular patterns of turbulent spots in a laminar boundary layer. In Proc. IUTAM Symp. on Laminar-Turbulent Transition (ed. R. Eppler \& H. Fasel), pp. 227-287. Springer.

Cook, W. J. \& Felderman, E. J. 1966 Reduction of data from thin-film heat-transfer gauges: a concise numerical technique. AIAA J. 4, 561-562.

Doorly, D. J. \& SMith, F. T. 1992 Initial-value problems for spot disturbances in incompressible or compressible boundary layers. J. Engng Maths 26, 87-106.

Emmons, H. W. 1951. The laminar-turbulent transition in a boundary layer. Part 1. $1951 \mathrm{~J}$. Aeronaut. Sci. 18, 490-498.

Fernholz, H. H. \& Finley, P. J. 1977 A critical compilation of compressible turbulent boundary layer data. AGARDograph 223.

FISCHER M. C. $1972 a$ Turbulent bursts and rings on a cone in helium at $M=7.6$. AIAA J. 10, 1387-1388.

FisCHER, M. C. $1972 b$ Spreading of a turbulent disturbance. AIAA J. 10, 957-959.

Gad-el-Hak, M., Blackwelder, R. F. \& Riley, J. J. 1981 On the growth of turbulent regions in laminar boundary layers. J. Fluid Mech. 110, 73-95.

Gostelow, J. P., Hong, G. \& Sheppeard, M. A. 1992 Investigations of a by-pass turbulent spot with reference to boundary layers on turbomachinery blading. Proc. ISROMAC 4 Conf.

GutMark, E. \& BLACKWELder, R. F. 1987 On the structure of a turbulent spot in a heated laminar boundary layer. Exps. Fluids 5, 217-229.

Hillier, R., Boyce, R. R., Creighton, S. A., Fiala, A., Jackson, A. P., Mallinson, S. G., Sheikh, A. H., Soltani, S. \& Williams, S. 2003 Development of some hypersonic benchmark flows using CFD and experiment. Shock Waves 12, 375-384.

Hillier, R., Kirk, D. \& Soltani, S. 1995 Navier-Stokes computations of hypersonic flows. Intl J. Numer. Methods Heat Fluid Flow 5, 195-212.

Hong, G. 1995 Structure of an artificial turbulent spot in a transitional boundary layer. In Proc. AMSE FED, Industrial and Environmental Applications of Fluid Mechanics, vol. 221, pp. 33-38.

JACKSON, A. P., Hillier, R. \& Soltani, S. 2001 Experimental and computational study of laminar cavity flows at hypersonic speeds. J. Fluid Mech. 427, 329-358.

JAMES, C. S. 1958 Observation of turbulent-burst geometry and growth in supersonic flow. NACA TN 4235.

Keyes, F. G. 1952 The heat conductivity, viscosity, specific heat and Prandtl numbers for thirteen gases. Massachussetts Institute of Technology, Project Squid, Tech. Rep. 37.

Kimmel, R. L. 1997 The effect of pressure gradients on transition zone length in hypersonic boundary layers. J. Fluids Engng 119, 36-41.

LAderman, A. J. 1980 Adverse pressure gradient effects on supersonic boundary-layer turbulence. AIAA J. 18, 1186-1195. 
Mallinson, S. G., Hillier, R., Jackson, A. P., Kirk, D. C., Soltani, S. \& Zanchetta, M. 2000 Gun tunnel flow calibration: defining input conditions for hypersonic flow computations. Shock Waves 10, 313-322.

Matsui, T. 1980 Visualization of turbulent spots in the boundary layer along a flat plate in a water flow. In Proc. IUTAM Symp. on Laminar-Turbulent Transition (ed. R. Eppler \& H. Fasel), pp. 288-296. Springer.

MeE, D. J. 2001 Transition measurements on a 5 degree cone in the T4 shock tunnel. University of Queensland Res. Rep. 2001-2.

MeE, D. J. 2002 Boundary-layer transition measurements in hypervelocity flows in a shock tunnel. AIAA J. 40, 1542-1548.

Mee, D. J. \& Goyne, C. P. 1996 Turbulent spots in boundary layers in a free-piston shock tunnel flow. Shock Waves 6, 337-343.

Nagamatsu, H. T., Sheer, R. E. \& Graber, B. C. 1967 Hypersonic laminar boundary layer transition on 8-foot-long, 10 degree cone, $M=9.1-16$. AIAA J. 7, 1245-1252.

Narasimha, R., Subramanian, C. \& Badri Narayanan, M. A. 1984 Turbulent spot growth in favorable pressure gradients. AIAA J. 22, 837-839.

Perry, A. E., Lim, T. T. \& TeH, E. W. 1981 A visual study of turbulent spots. J. Fluid Mech. 104, $387-405$.

Rathnasingham, R. \& BreueR, K. S. 1997 System identification and active control of a turbulent boundary layer. AIAA Paper 97-1793.

REDA, D. C. 1977 Boundary-layer transition experiments on sharp, slender cones in supersonic free flight. NSWC/NOL TR 77-59.

REDA, D. C. 1979 Boundary-layer transition experiments on sharp, slender cones in supersonic free flight. AIAA J. 17, 803-810.

Riley, J. J. \& Gad-el HaK, M. 1985 The dynamics of turbulent spots. In Frontiers in Fluid Mechanics (eds. S. Davies \& J. Lumley), pp. 123-155. Springer.

Sankaran, R., Antonia, R. A., Bisset, D. L. \& Sokolov, M. 1991 Flow patterns and organization within a turbulent spot. Phys. Fluids A 3, 1560-1571.

SchneIder, S. P. 2004 Hypersonic laminar-turbulent transition on circular cones and scramjet forebodies. Prog. Aerospace Sci. 40, 1-50.

Schubauer, G. B. \& Klebanoff, P. S. 1955 Contributions on the mechanics of boundary layer transition. NACA TN 3489.

Schultz, D .L. \& Jones, T. V. 1973 Heat-transfer measurements in short-duration hypersonic facilities. AGARDograph 165.

Seifert, A. \& Wygnanski, I. J. 1995 On turbulent spots in a laminar boundary layer subjected to a self-similar adverse pressure gradient. J. Fluid Mech. 296, 185-209.

Stetson, K. F. 1988 On cone frustrum pressure gradient effects on transition. AIAA J. 26, 500-502.

Stetson, K. F. \& Rushton, G. H. 1967 Shock tunnel investigation of boundary layer transition at $M=5.5$. AIAA J. 5, 899-906.

Wygnanski, I., Sokolov, M. \& Friedman, D. 1976 On a turbulent 'spot' in a laminar boundary layer. J. Fluid Mech. 78, 785-819.

Zanchetta, M. A. 1996 Kinetic heating and transition studies at hypersonic speeds. PhD thesis, University of London.

Zanchetta, M. A. \& Hillier, R. $1996 a$ Boundary layer transition on slender blunt cones at hypersonic speeds. In Proc. 20th Intl Symp. on Shock Waves (ed. B. Sturtevant, J. Shepherd \& H. Hornung), pp. 699-704. World Scientific.

ZanchetTA, M. A. \& Hillier, R. $1996 b$ Blunt cone transition at hypersonic speeds: the transition reversal regime. In Transitional Boundary Layers in Aeronautics (ed. R. A. W. M. Henkes \& J. L. van Ingen), pp. 433-440. North-Holland. 